\title{
Morphometric Analysis and Flash Floods Assessment for Drainage Basins of the Ras En Naqb Area, South Jordan Using GIS
}

\author{
Yahya Farhan, Omar Anaba, Ali Salim \\ Department of Geography, University of Jordan, Amman, Jordan \\ Email: yahyafarhan2100@outlook.com
}

Received 27 April 2016; accepted 4 June 2016; published 7 June 2016

Copyright (C) 2016 by authors and Scientific Research Publishing Inc.

This work is licensed under the Creative Commons Attribution International License (CC BY). http://creativecommons.org/licenses/by/4.0/

(c) (i) Open Access

\section{Abstract}

Morphometric analysis and flash floods assessment were conducted for the watersheds of Ras En Naqb escarpment, south Jordan. The study area comprises of twelve small watersheds occupying the faulted-erosional slopes, and the dip slopes. The drainage network shows that dendritic and sub-dendritic patterns dominated the dip slopes, whereas trellis pattern characterized the faultederosional slopes. Stream orders range from fourth to sixth order. The mean bifurcation ratios vary between 4.2 and 5.38 for the dip slope basins, and between 3.5 and 5.0 for the faulted-erosional slope watersheds, indicating a noticeable influence of structural disturbances (i.e., faulting and uplifting), and rejuvenation of drainage networks. All watersheds have short basin lengths, ranging from $23.8 \mathrm{~km}$ to $42.2 \mathrm{~km}$ for the dip slope basins, and between $15.3 \mathrm{~km}$ and $45.4 \mathrm{~km}$ for the faulted-erosional slope catchments. This is indicative of high flooding susceptibility associated with heavy rainstorms of short duration. The circularity ratios range from 0.177 to 0.704 which denote that the catchments are moderately circular on the faulted-erosional slopes, and to some extent elongated on the dip slopes. The length of overland flow values ranges from 0.854 to 0.924 for the dip slope catchments, whereas $L_{0}$ values for the faulted-erosional slopes vary from 0.793 to 0.945 denoting steep slopes and shorter paths on both dip slope and faulted-erosional slope watersheds. Values of stream frequency range from 1.509 to 1.692 for the dip slope, and from 1.688 to 2.0 for the faulted-erosional slope catchments. $F_{S}$ values are also indicative of slope steepness, low infiltration rate, and high flooding potential. The watersheds of the dip slopes show lower values of form factor varying from 0.079 to 0.364 , indicating elongated shape and suggesting a relatively flat hydrograph peak for longer duration. Similarly, values of $D_{d}$ are high for catchments on the dip slope basins (1.709 - 1.85) and the faulted-erosional slope watersheds $(1.587-2.0)$ indicating highly dissected topography, high surface runoff, low infiltration rate, and consequently high flooding potential. Furthermore, high relief values exist, ranging from $388 \mathrm{~m}$ to $714 \mathrm{~m}$ for the dip slope basins, and from $421 \mathrm{~m}$ to $846 \mathrm{~m}$ for the faulted-erosional slope catchments indicting high relief and steep slopes. Morphometric analysis, and flash flood assessment suggest that ten watersheds (83.3\%) are categorized under high and intermediate flooding susceptibility, and the 
faulted-erosional slope catchments are more hazardous in terms of flooding. Thus the protection of Ma'an, El Jafr rural Bedouin settlements, and Amman-Aqaba highway from recurrent flooding is essential to ensure sustainable future development in Ras En Naqb-Ma'an area.

\title{
Keywords
}

\author{
Ras En Naqb Escarpment, Flash Flood Assessment, Dip Slopes, Faulted-Erosional Slopes, \\ Hypsometric Integral, Jordan
}

\section{Introduction}

Drainage basins represent fundamental geomorphic units for hydrological management and sustainable natural resources development. Geological and morphological setting, topography, and climate constitute major physical factors controlling the geometry of fluvial system, drainage systems and density. Variations in physical conditions occasionally resulted in variations of morphometric characteristics of drainage basins and the associated fluvial systems [1]. Detailed morphometric analysis of watersheds, helps to explore the geomorphic history, evolution and characteristics of landforms, and development of drainage networks. Changes in the fluvial dynamics caused by natural components or anthropogenic intervention often resulted in morphological changes across drainage basins. The physical properties of drainage basins (i.e., shape, size, drainage density, length and size of streams) are correlated remarkably with the hydrological parameters characterized drainage basins [2]-[6]. Since the Second World War, substantial research developed and focused on elaborating morphometric parameters, their computation, morphometric analysis methods, and characterization of drainage basins and stream networks. Quantitative methods have been utilized in morphometric analysis of drainage basins initially by [7]-[11], whereas, multivariate statistical techniques were employed earlier by Mather and Doornkamp [12]. Delineation of drainage networks within a catchment or sub-catchment was achieved traditionally based on field observation, topographic maps (Scale 1:25,000 and 1:50,000) and air photos. Recently, geospatial analytical techniques (GIS and remote sensing) have been developed and employed as powerful tools for computation, quantitative description and assessment of morphometric parameters, thematic mapping of morphometric variables, and the application of morphometric analysis in different fields of research such as: hydrology and appraisal of environmental hazard [13]-[16]. Geospatial tools have dramatically reduced the time and cost of analysis, and can be applied easily in both mountainous, highly dissected/rugged topography, and in accessible areas. Geology (lithology and tectonics), morphology (topography and slopes), and climate are considered the major attributes which determine the characteristics and evolution of drainage basins and drainage networks. The fluvial system and the associated hydro-geomorphic processes are decisive for watershed hydrology, mainly surface water resources. Morphometric analysis of drainage basin geometry provides essential information which helps to understand geomorphic evolution of drainage basins, landforms and slopes development [7] [9] [11], and for demarcating erosion-prone areas including landslide complexes. Such information is vital for watershed prioritization for soil and water conservation, and water resources management. Morphometric analysis also helps to infer the hydrological characteristics of drainage basins; therefore, it facilitates hydrological prospecting, assessment of the potential of groundwater recharge, and mapping of flood prone areas. The present work describes the results of morphometric analysis of twelve mountainous/arid watersheds in the Ras En Naqb area, south Jordan to understand watersheds behavior with respect to flash floods using ASTER DEM and GIS. The study intends to illustrate the procedure of assessing drainage network, basin geometry, drainage texture analysis, and relief morphometric parameters using geoprocessing tools provided by Arc GIS software. The potential of ASTER DEM data and GIS in drainage basin morphometry was verified in comparison with conventional methods. The use of ASTER DEM and GIS also enables rapid, precise, and inexpensive tools to extract and analyze morphometric data for flash floods assessment and other applications such as hydrology, prioritization of watersheds for soil and water conservation [17]-[21]. The significance of morphometric analysis of these wadis is justified by the fact that some of the dip slope catchments occasionally threatened Ma'an city earlier with severe flash floods, and inundation of the El Jafr depression during flooding. Similarly, the flash floods of the faulted-erosional slope wadis (with steep slopes), occasionally caused serious inundation of the playas east 
of Quweira town; this disrupts the movements of the Bedouin (with their camels and goats), and the tourists moving around the picturesque inselberg landscape. Bifurcation ratio, drainage density, and stream frequency parameters have been employed recently to assess flash flooding susceptibility in arid watersheds of the Middle East [22].

\section{Study Area}

The Ras En Naqb escarpment covers an area of approximately $566 \mathrm{~km}^{2}$, and is located between $35^{\circ} 28^{\prime} \mathrm{E}$ to $35^{\circ} 83^{\prime} \mathrm{E}$ longitude, and $29^{\circ} 47^{\prime} \mathrm{N}$ to $30^{\circ} 06^{\prime} \mathrm{N}$ latitude (Figure 1). It separates the high flat Central Jordan Plateau (1500 - $1600 \mathrm{~m}$ a.s.l), from the rugged inselbergs to the south, at an elevation ranging from 700 to $800 \mathrm{~m}$ a.s.l [23]. Earlier, deVaumas [24] identified the Ras En Naqb escarpment as a huge cuesta striking WNW with a height ranging between 200 and $400 \mathrm{~m}$. Parker [25] describes the escarpment as an erosional feature, where fluvial erosion has been activated along a number of sub-parallel NW-trending faults affecting the area [26]. The development of Ras En Naqb escarpment is significantly affected by the lithological characteristics and bedding of sandstones and the overlying Cretaceous limestones, faulting and jointing. It is stated that most of NWtrending faulting occurred in the Late Miocene and possibly earlier [27] [28], and the Cretaceous limestone cover was extended much further south. Thus, the present escarpment is probably purely erosional. However, the Cretaceous rim itself and the water divide (separating the dip slope catchments from the faulted-erosional slope watersheds) are straight in a gross sense as illustrated by a clear linear edge in 3D perspective (Figure 2(a) and Figure 2(b)), elevation/hypsometry, aspect and slope patterns of the area (Figure 3 and Figure 4). Therefore, significant erosional irregularities are apparent from the Cretaceous rim down to the sandy desert floor and inselberg landscape. The drop from crest line (rim) to floor is interrupted by a prominent rocky bench developed on relatively resistant sandstones. The broad bench was later dissected by fluvial processes, and faulting, and has been split into irregular mesas with sharp and steep cliffs such as Jebel Rabigh. Close to Ras En Naqb railway station, the escarpment crest reached an elevation of $1573 \mathrm{~m}$ a.s.l, but eastwards, its elevation decreases to 1110 $\mathrm{m}$ a.s.l near Batn el Ghol station. The noticeable height difference between the western and eastern part of the escarpment is attributed to erosion. The eastern part of the escarpment is highly eroded and subdued, thus converted to a fault-line scarp [23]. The sandstone inselberg landscape has been formed as a result of fluvial dissection of Paleozoic sandstones following the stripping of Cretaceous, Tertiary Carbonates by erosion and weathering. This process was combined with graben-faulting, depositional infilling, and arid-climate slope retreat, all of which produced a relatively flat plain which accommodates erosional residuals [28]. The granite horst to the west is separated from the sandstone inselbergs and Ras En Naqb escarpment by a normal fault which runs just west of Quweira town, with a down throw to the east of $\approx 600 \mathrm{~m}$ [29]. The elevation of the flat sandy desert floor rises from 750 m a.s.l at Qa (playa) Um Salab to about $1200 \mathrm{~m}$ a.s.l at the base of Ras En Naqb escarpment. Here, the cliffed sandstone inselbergs are characteristic. The inselberg landforms are developed mainly in

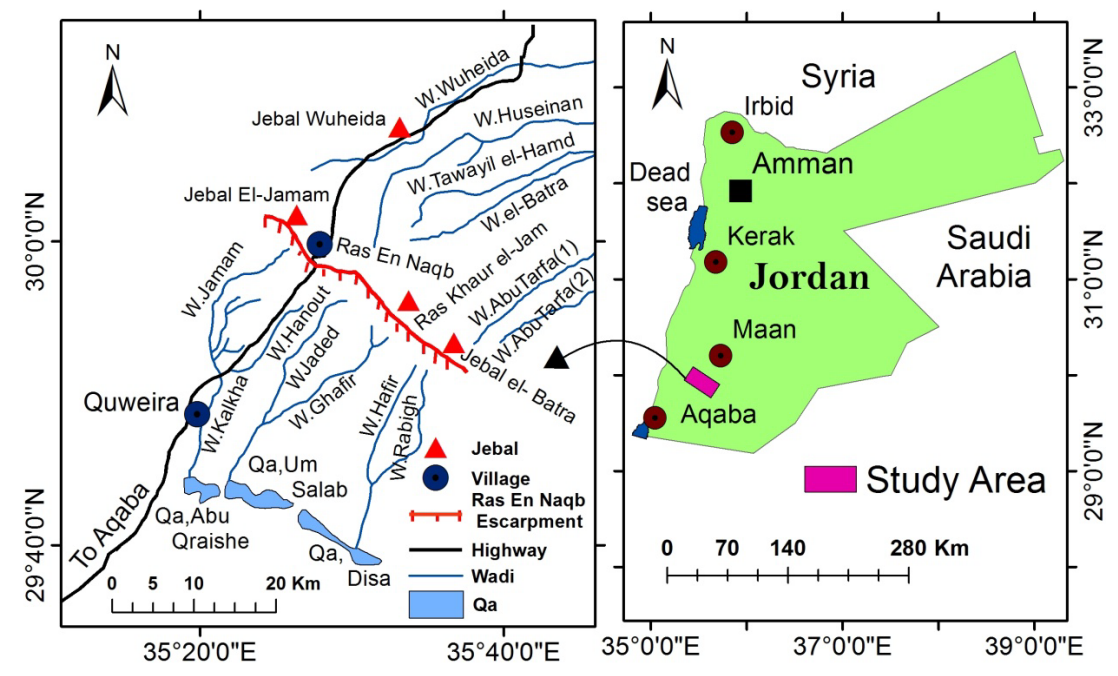

Figure 1. The study area. 


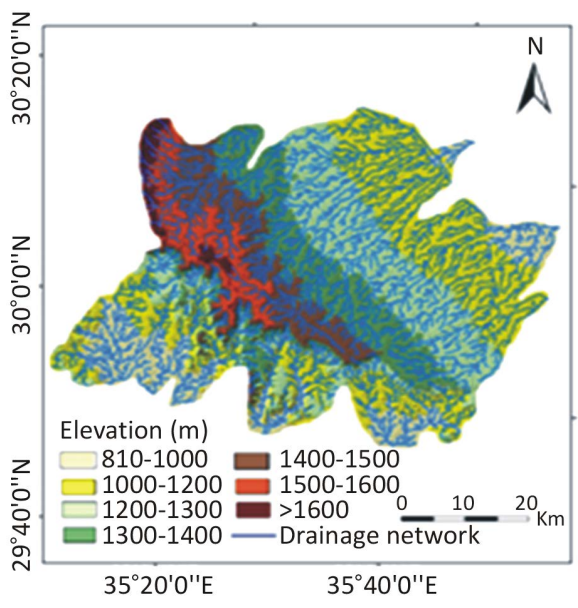

(a)
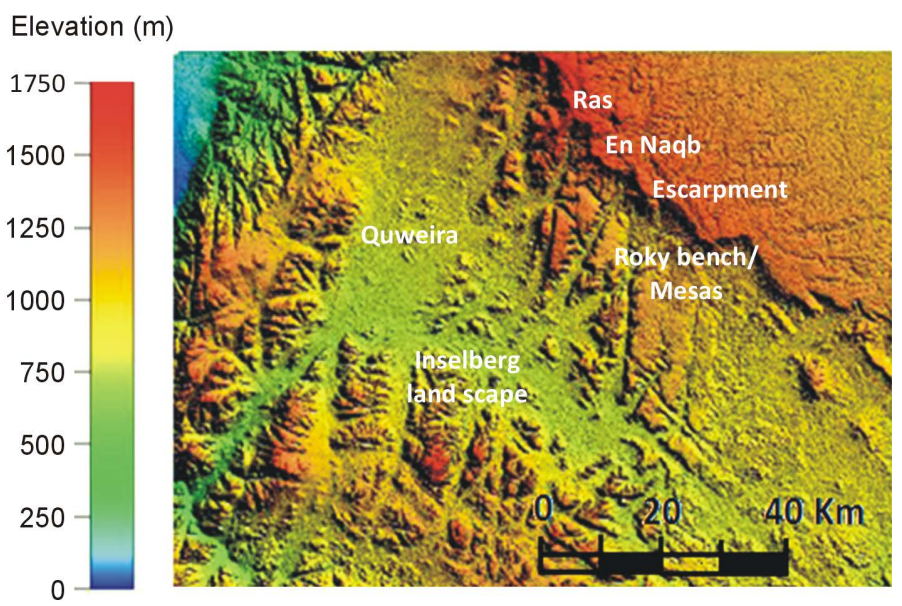

(b)

Figure 2. (a) and (b) 3D perspective for Ras En Naqb area.

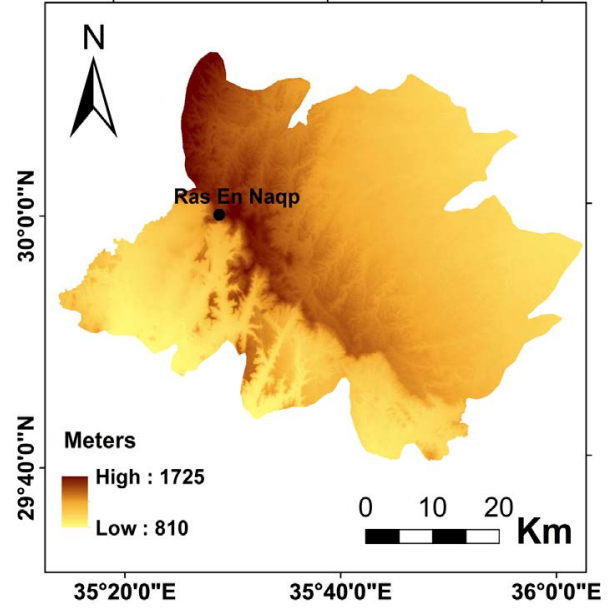

(a)

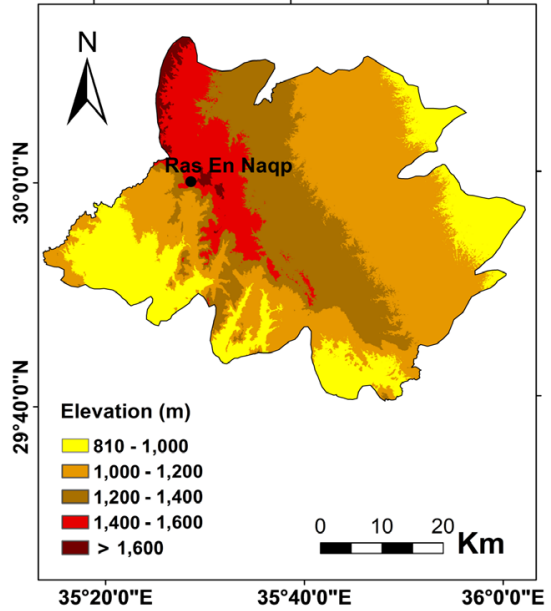

(b)

Figure 3. The digital elevation model (a) and hypsometry (b) of Ras En Nagb elevation.

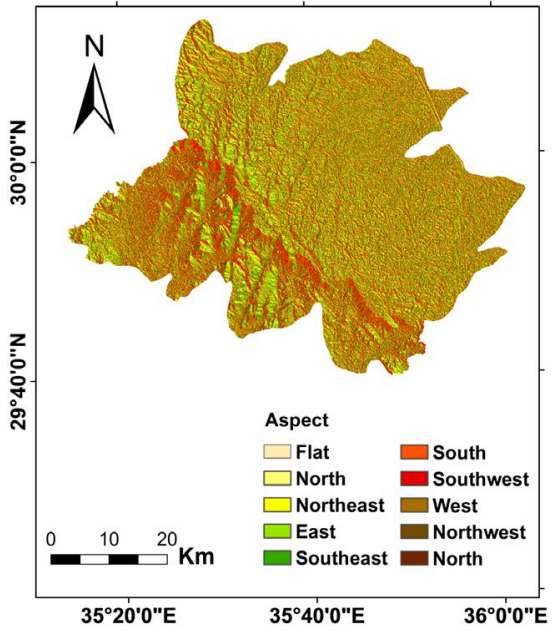

(a)

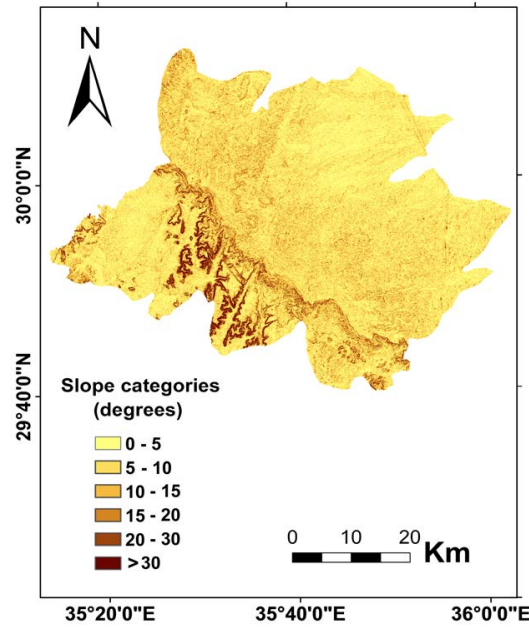

(b)

Figure 4. Aspect (a) and slope categories (b) of Ras En Naqb area. 
Cambrian, Ordovisian and lower Cretaceous Kurnub sandstones and shale (Figure 5) [30] [31]. The base level of the catchment draining the faulted-erosional slopes comprises three playas occupying a NW-trending depression. These are: Qa Abu Qrishi, Qa Um Salab and Qa Disi. The linear aspect of the depression demonstrates its structural origin. The dip slopes east of Ras En Naqb, grade eastwards into a gentle-flat, stony hamada surface covered by plateau gravel from the weathering of the underlying parent rocks. The thickness of these covers increases near the margins of El Jafr depression. The hamada landscape is well developed as a Reg surface which covers hundreds of square kilometers east of Ma'an city. It is paved by stony covers and polished dark brown by desert patina.

Recurrent landslide movement is characteristic of Ras En Naqb escarpment, and occasionally threatened the Amman-Aqaba highway. Oversteepening of slopes due to fluvial erosion, differential weathering, and steep cut slopes enhance rock falls and rock slides from heavily jointed limestone beds. Percolation of water from relatively abundant rainfall over Ras En Naqb (140 mm of annual rainfall/snow), and the repetitive heavy rainstorms towards impervious marl, shale and clay beds of the nodular limestone unit reduce the cohesion of the materials, thus encouraging landslide activity. The dip slope of the Ras En Naqb escarpment is composed of lower Cretaceous marl, shale and chalk, sloping $3^{\circ}$ to $5^{\circ}$ NNE towards Ma'an. A number of monoclinal flexures exist here instead of faulting, and graben structures dominate a considerable part of the escarpment (Figure 5). Despite the slight gradient, all wadi courses crossing the dip slopes are currently cutting down deeper channels through the alluvial fill as a response to the lowering of the base level at El Jafr synsedimentary depression [23] [26]. The present study comprises six small catchments draining the dip slope into El Jafr depression to the northeast, and six catchments draining the faulted-erosional slopes towards the smaller playas to the southwest (Figure 6). The climate is cold dry arid on the crest of Ras En Naqb escarpment, and hot dry arid in the Quweira-Qa'Disi area. Due to the variation in topography, the average annual rainfall varies from $39.9 \mathrm{~mm}$ at Ma'an (1006 $\mathrm{m}$ a.s.l) to $140 \mathrm{~mm}$ at Ras En Naqb village (1573 m a.s.l). It is postulated [29] that the wadis of Ras En Naqb area have about five to seven run-off producing storms per year. Storms generally last no more than a few hours, but are often quite intense and repetitive [32] [33]. Average monthly temperatures range from $30^{\circ} \mathrm{C}+$ (summer maximum) to $\approx 7^{\circ} \mathrm{C}$ (winter minimum). However, freezing temperatures were recorded in January and February at Ras En Naqb and Ma'an. The low amount and sparse rainfall result in poor/sparse vegetation, which consists of scattered tamarisk, acacia and cenapod, and some annual grasses. The land use/land cover is restricted to Ras En Naqab village, scattered and small patches of olive trees and woods. Thus, most of the area is considered poor rangeland and bare lands (mostly hamada surface) (Figure 7). The Ras En Naqb escarpment represents an unstable

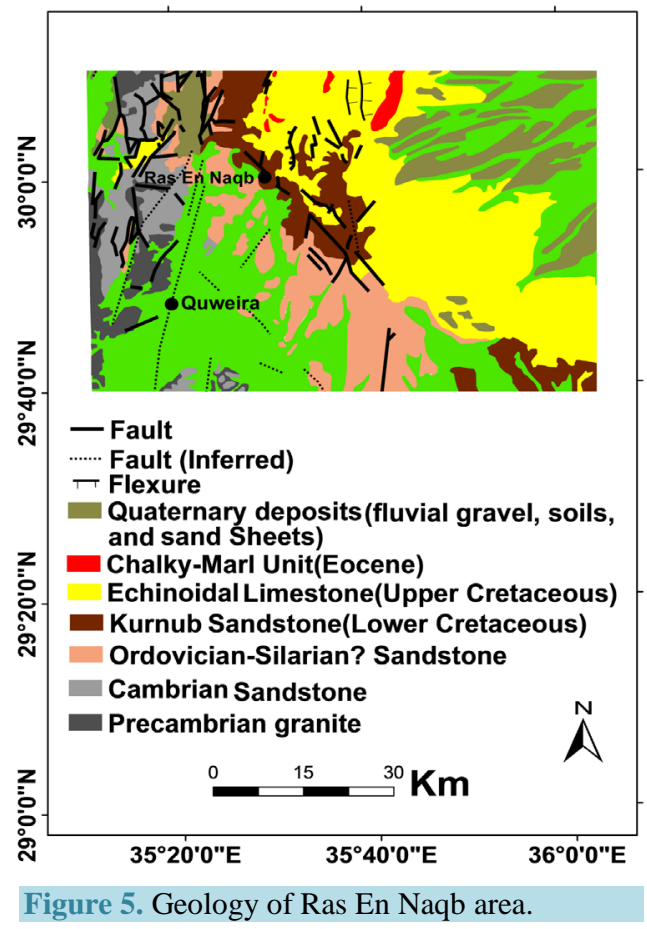




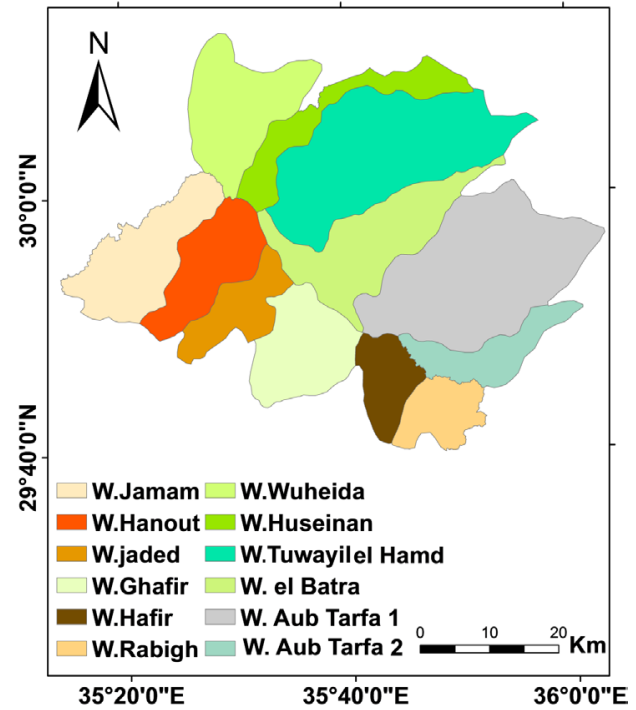

Figure 6. The watersheds of Ras En Naqb escarpment.

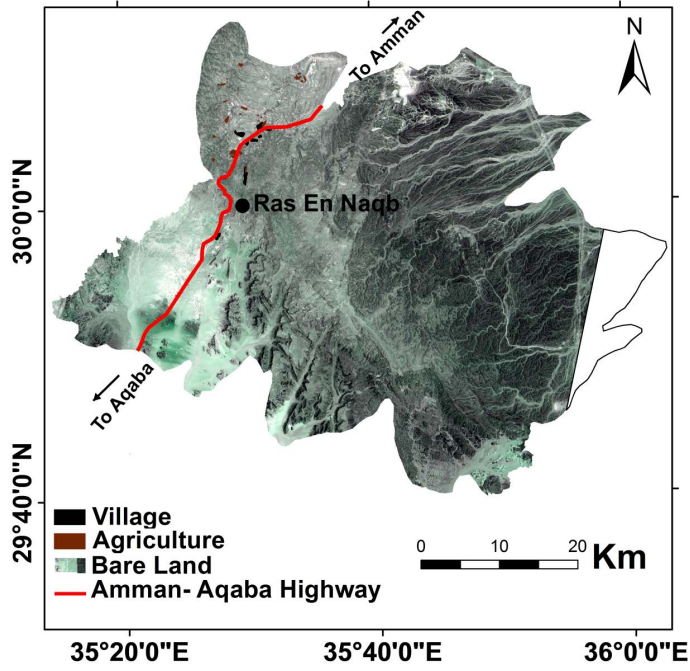

Figure 7. Land use/land cover in Ras En Naqb area (most of the area is bare land).

morphological unit due to the presence of soft rock units, i.e. the Kurnub sandstone and shale, and the lower Cretaceous limestone, marl and shale. These rock units are heavily faulted and jointed. Along with recurrent intense rainstorms, they are deemed a major factor influencing slope instability. Old and fresh landslide scars exist, which indicate that landsliding is an active process at present, and threatens the Amman-Aqaba highway once every few years.

\section{Methodology}

Morphometric analysis for Ras En Naqb watersheds was conducted using topographic maps with a scale 1:50,000 (20 m contour interval), ASTER DEM, and Arc GIS 10.1 software package. ASTER DEM is provided on line cost free to all researchers, and is available in Geo Tiff format, with geographic latitude/longitude coordinates at 1 arc-second, approximately $30 \mathrm{~m}$ grid cell size. The elevation error of the DEM is reported to be +7.4 $\mathrm{m}$ for forest land cover, and $-0.7 \mathrm{~m}$ for bare land and complex terrain resembling the Ras En Naqb area. Furthermore, the horizontal error is stated to be an East/West shift of -0.13 arc-seconds and North/South shift of 
0.19 arc-seconds when compared to the 10-m mesh DEM produced by the Geographical Survey Institute (GIS) of Japan [34] [35]. Recent studies were carried out on the earth's lowest elevation: the Dead Sea (Jordan) to test the validity of ASTER DEM [36], and to compare ASTER DEM, and SRTM (the Shuttle Radar Topographic Mission) DEM against a referenced DEM constructed from 1:25,000 scale topographic map [37]. It is reported that the overall accuracy of the DEM is in line with the reported official accuracy specifications [36]. The comparison also reveals that SRTM overestimates and ASTER DEM underestimates the elevations. In this regard, SRTM DEM is comparatively more accurate than ASTER DEM, but with $90 \mathrm{~m}$ resolution, whereas, ASTER DEM is of $30 \mathrm{~m}$ spatial resolution. However, both DEMS are employed heavily at present to delineate watershed and sub-watershed boundaries, and drainage networks, to derive and calculate drainage morphometric information, and to establish cross sections [37]. Considering the Ras En Naqb area, which consists mostly of bare land and arid climate, along with the availability of ASTER DEM (30 m spatial resolution), and the Arc GIS tools, both were employed to derive, and calculate the morphometric parameters of the Ras En Naqb watersheds. Different terrain feature/maps for the study area such as aspect, slope categories, elevation were generated using the Spatial Analyst module. Topo sheets were used initially to demarcate the boundaries of the watersheds, then, the ArcHydro tool was utilized to delineate the final watershed boundaries and stream networks for the twelve catchments. The stream order maps were compiled from the flow direction map for each watershed using Stream Order tool. The stream ordering system used was based on Strahler's method [3] [10] [38]. Basic morphometric parameters such as: area $(A)$, basin length $\left(L_{b}\right)$, perimeter $(P)$, stream order $(\mathrm{u})$, stream number $\left(\mathrm{N}_{\mathrm{u}}\right)$, stream length $\left(\mathrm{L}_{\mathrm{u}}\right)$, were measured directly from the DEM using GIS software. Other parameters including bifurcation ratio $\left(\mathrm{R}_{b}\right)$, drainage density $\left(\mathrm{D}_{\mathrm{d}}\right)$, drainage frequency $\left(\mathrm{F}_{\mathrm{s}}\right)$, length of overland flow $\left(\mathrm{L}_{0}\right)$, circularity ratio $\left(R_{c}\right)$, elongation ratio $\left(R_{e}\right)$, basin relief $\left(B_{h}\right)$, relief ratio $\left(R_{r}\right)$, form factor $\left(R_{f}\right)$, and shape factor $\left(B_{s}\right)$ were calculated based on mathematical equations illustrated in Table 1 . The hypsometric curve $\left(\mathrm{C}_{c}\right)$ and hypsometric integral $\left(\mathrm{H}_{\mathrm{i}}\right)$ were prepared earlier using topo sheets at a scale 1:50,000 [39].

To assess flash floods potential for the Ras En Naqb watersheds, El-Shamy's approach was adopted [22]. It is a simple morphometric method which has been designated to estimate flash flood risk levels and the degree of hazardousness for each watershed. Two different approaches were elaborated to determine the hazardous catchment. The first is based on the relationship between bifurcation ratio $\left(R_{b}\right)$ and drainage density $\left(D_{d}\right)$ whereas the second approach employed the relationship between bifurcation ratio $\left(R_{b}\right)$ and stream frequency $\left(F_{s}\right)$. Drainage density $\left(D_{d}\right)$ refers to relief dissection, runoff potential, infiltration capacity of surface materials, climate, and land cover of the watershed. Accordingly, low values of $D_{d}$ indicate optimal conditions of infiltration, thus decreasing runoff potential, while, high stream frequency $\left(\mathrm{F}_{\mathrm{s}}\right)$ represents impermeable sub-surface materials, poor vegetation cover, high relief, and low infiltration capacity, thus, increasing runoff potential [3] [38].

Applying, this relationship separately to each catchment, will provide reasonable estimation on flooding risk, and groundwater aquifer recharge. The resultant illustrations for $D_{d} v s . R_{b}$ and $F_{s} v s . R_{b}$ have to be plotted graphically, where each illustration contains two curves dividing the area into three zones which can be described as follows:

- Zone A represents low probability of floods and high groundwater aquifer recharge.

- Zone B refers to catchments with intermediate possibility of floods and moderate potential for groundwater aquifer recharge.

- Zone C indicates high possibility of floods and low recharge potential.

If a watershed has two different fields, then the appropriate classification plot has been selected.

\section{Results and Discussion}

\subsection{Morphometric Analysis}

Quantitative analysis of the 12 watersheds developed on Ras En Naqb escarpment was implemented based on 23 morphometric variables which represents drainage network, geometry, texture and relief aspects of the catchment. The drainage pattern is dendritic to sub-dendritic type on dip slopes, whereas, trellis pattern dominates the faulted-erosional slopes. In the present study, stream ordering for the Ras En Nagb watersheds has been ranked according to Strahler's method of the hierarchical ranking system [3] [38].

The calculated morphometric parameters are illustrated in Table 2 and Table 3, and will be discussed accordingly. Based on drainage order, all the catchments of faulted-erosional slopes are all of fifth order. Three watersheds of the dip slopes can be classified as three of sixth order. Two of fourth order, and one basin is of fifth 
Table 1. Morphometric parameters and their mathematical formula.

\begin{tabular}{|c|c|c|}
\hline Morphometric parameters & Formula/definition & References \\
\hline \multicolumn{3}{|l|}{ I. Drainage network } \\
\hline 1) Stream order (u) & Hierarchical rank & [10] \\
\hline 2) No. of streams $\left(\mathrm{N}_{\mathrm{u}}\right)$ & $\mathrm{N}=\mathrm{N}_{1}+\mathrm{N}_{2}+\cdots+\mathrm{N}_{\mathrm{n}}$ & [7] \\
\hline 3) Stream length $\left(L_{u}\right) \mathrm{km}$ & $\mathrm{Lu}=\mathrm{L}_{1}+\mathrm{L}_{2}+\cdots+\mathrm{L}_{\mathrm{n}}(\mathrm{km})$ & [3] \\
\hline 4) Mean stream length $\left(L_{s m}\right) \mathrm{km}$ & $\mathrm{L}_{\mathrm{sm}}=\mathrm{L}_{\mathrm{u}} / \mathrm{N}_{\mathrm{u}}(\mathrm{km})$ & [3] \\
\hline 5) Stream length ratio $\left(R_{L}\right)$ & $\begin{array}{l}R_{L}=L_{u} / L_{u-1} \text {, where } L_{u}=\text { the total stream length of order } \\
\text { " } u \text { ", } L_{u-1}=\text { the total stream length of its next lower order }\end{array}$ & [7] \\
\hline 6) Bifurcation ratio $\left(\mathrm{R}_{\mathrm{b}}\right)$ & $\begin{array}{c}R_{b}=N_{u} / N_{u+1} \text {, where } N_{u}=\text { total no. of stream segments of order } \\
\text { "u", } N_{u+1}=\text { no. of segments of the next higher order }\end{array}$ & [8] \\
\hline 7) Mean bifurcation ratio $\left(\mathrm{R}_{\mathrm{bm}}\right)$ & $\mathrm{R}_{\mathrm{bm}}=$ average of bifurcation ratio of Strahler all orders & [10] \\
\hline \multicolumn{3}{|l|}{ II. Basin geometry } \\
\hline 8) Basin length $\left(\mathrm{L}_{\mathrm{b}}\right) \mathrm{km}$ & Length of the basin $(\mathrm{km})$ & {$[7]$} \\
\hline 9) Basin area (A) km² & Plan area of the watershed $\left(\mathrm{km}^{2}\right)$ & [7] \\
\hline 10) Basin perimeter $(\mathrm{P}) \mathrm{km}$ & Perimeter of the watershed $(\mathrm{km})$ & {$[7]$} \\
\hline 11) Form factor (ratio) $\left(R_{f}\right)$ & $\mathrm{R}_{\mathrm{f}}=\mathrm{A} / \mathrm{L}_{\mathrm{b}}^{2}$ & [7] \\
\hline 12) Elongation ratio $\left(R_{e}\right)$ & $\mathrm{R}_{\mathrm{e}}=1.128 \sqrt{\mathrm{A}} / \mathrm{L}_{\mathrm{b}}$ & [8] \\
\hline 13) Shape factor $\left(B_{s}\right)$ & $\mathrm{B}_{\mathrm{s}}=\mathrm{L}_{\mathrm{b}}^{2} / \mathrm{A}$ & [7] \\
\hline 14) Lemniscate ratio $(\mathrm{k})$ & $K=L^{2} / 4 A$ & [48] [49] \\
\hline 15) Circularity ratio $\left(R_{c}\right)$ & $\mathrm{R}_{\mathrm{c}}=4 * \pi * \mathrm{~A} / \mathrm{P}^{2}$ & [3] [50] \\
\hline 16) Drainage texture $\left(D_{t}\right)$ & $\begin{array}{c}D_{t}=N_{u} / P \text {, where } N_{u}=\text { Total no. Streams of all orders, } \\
P=\text { perimeter }(\mathrm{km})\end{array}$ & [7] \\
\hline \multicolumn{3}{|l|}{ III. Drainage texture analysis } \\
\hline 17) Stream frequency $\left(F_{s}\right)$ & $\mathrm{F}_{\mathrm{s}}=\mathrm{N}_{\mathrm{u}} / \mathrm{A}$ & [7] \\
\hline 18) Drainage density $\left(D_{d}\right) \mathrm{km} / \mathrm{km}^{2}$ & $\mathrm{D}_{\mathrm{d}}=\mathrm{L}_{\mathrm{u}} / \mathrm{A}$ & [7] \\
\hline 19) Drainage intensity $\left(D_{i}\right)$ & $\mathrm{D}_{\mathrm{i}}=\mathrm{F}_{\mathrm{s}} / \mathrm{D}_{\mathrm{d}}$ & {$[55]$} \\
\hline 20) Length of overland flow $\left(L_{0}\right) \mathrm{km}$ & $\mathrm{L}_{\mathrm{o}}=1 / 2 \mathrm{D}_{\mathrm{d}}$ & [7] \\
\hline \multicolumn{3}{|l|}{ IV. Relief characteristics } \\
\hline 21) Basin relief $\left(B_{h}\right)$ or total relief $(H) m$ & $\begin{array}{c}\mathrm{B}_{\mathrm{h}}=\mathrm{h}-\mathrm{h}_{1} \text {, where, } \mathrm{h}=\text { maximum height }(\mathrm{m}) \text {, } \\
\mathrm{h}_{1}=\text { minimum height }(\mathrm{m})\end{array}$ & [9] \\
\hline 22) Relief ratio $\left(\mathrm{R}_{\mathrm{r}}\right)$ & $\mathrm{R}_{\mathrm{r}}=\mathrm{H} / \mathrm{L}_{\mathrm{b}}$, Where $\mathrm{H}=$ total relief, $\mathrm{L}_{\mathrm{b}}=$ basin length & [3] \\
\hline 23) Ruggedness number $\left(R_{n}\right)$ & $\mathrm{R}_{\mathrm{n}}=\mathrm{D}_{\mathrm{d}} *\left(\mathrm{~B}_{\mathrm{h}} / 1000\right)$ & [3] \\
\hline 24) Dissection index $\left(D_{\text {is }}\right)$ & Dis $=B_{h} / R a$, where $R_{a}=$ absolute relief & {$[56]$} \\
\hline 25) Hypsometric curve (HC) & $\begin{array}{l}\text { HC is achieved by plotting the proportion of the total } \\
\text { height }(\mathrm{h} / \mathrm{H}) \text { against the proportion of the total area }(\mathrm{a} / \mathrm{A}) \\
\text { of the basin, where } \mathrm{H} \text { is the total relief height, a is the } \\
\text { total area of the basin above a given line of elevation } \mathrm{h} \text {. }\end{array}$ & [2] [3] [38] \\
\hline & $\mathrm{Hi}=(\overline{\mathrm{H}}-\mathrm{H}) /(\mathrm{H}-\mathrm{h})$, where & \\
\hline 26) Hypsometric integral (Hi) & $\begin{array}{c}\overline{\mathrm{H}}=\text { the weighted mean elevation } \\
\mathrm{H}=\text { maximum elevation } \\
\mathrm{h}=\text { minimum elevation }\end{array}$ & [2] [38] \\
\hline
\end{tabular}


order (Table 2 and Table 3; Figure 7 and Figure 8). Such variation indicates the influence of structure, lithology, morphology and slope steepness on drainage network development especially on the faulted-erosional slopes. By contrast, lithological uniformity and relative availability of rainfall(the annual rainfall increased from $57 \mathrm{~mm}$ at Quweira to the southwest, to $140 \mathrm{~mm}$ at Ras En Naqb station), and long dip slopes intensified erosional processes, thus stream length and area increased at a rate exceeding the rate of increase in stream number.

\subsubsection{Drainage Network}

The total number of streams $\left(\mathrm{N}_{\mathrm{u}}\right)$ for the 12 watersheds is 2096, and the first order streams account for $79.3 \%$ of the total number of streams in all basins. The details of stream characteristics are ascertained by Horton's first law [7], the "law of stream number", which states that the number of streams of different orders in a given drainage basin tends to closely approximate an inverse geometric ratio. This inverse geometric relationship is shown graphically in the form of a straight line when $\log$ values $\mathrm{N}_{\mathrm{u}}$ are plotted on an ordinary graph (Figure 9(a)). It is also recognized that the number of streams gradually decreases as the stream order increases.

1) Stream length $\left(L_{u}\right)$ is a significant hydrological property and indicative of runoff characteristics, geomorphic development of stream segments, and tectonic instability. Generally, the higher the order, the longer the length of stream in nature. The steam length has been calculated according to the law elaborated by [7]. The total stream length varies between $168.939 \mathrm{~km}$ to $421.253 \mathrm{~km}$ for the catchments of faulted-erosional slopes, and between $204.957 \mathrm{~km}$ and $881.136 \mathrm{~km}$ for the basins of the dip slopes (Table 4 and Table 5). The first order

Table 2. Morphometric characteristics of faulted-erosional slope watersheds.

\begin{tabular}{|c|c|c|c|c|c|c|c|}
\hline Par. No. & Morphometric parameters & W.Jamam & W.Hanout & W.Jaded & W.Ghafir & W.Hafir & W.Rabigh \\
\hline 1 & Stream order (u) & $\mathrm{V}$ & $\mathrm{V}$ & $\mathrm{V}$ & $\mathrm{V}$ & $\mathrm{V}$ & $\mathrm{V}$ \\
\hline 2 & No. of streams $\left(\mathrm{N}_{\mathrm{u}}\right)$ (Total) & 405 & 345 & 193 & 333 & 166 & 180 \\
\hline 3 & Stream length $\left(\mathrm{L}_{\mathrm{u}}\right)$ (Total) Km & 421.253 & 302.434 & 181.362 & 321.742 & 168.939 & 169.140 \\
\hline 4 & Mean stream length $\left(\mathrm{L}_{\mathrm{sm}}\right)(\mathrm{km})$ & 1.040 & 0.876 & 0.939 & 0.966 & 1.017 & 0.939 \\
\hline 5 & Mean bifurcation ratio $\left(\mathrm{R}_{\mathrm{bm}}\right)$ & 3.752 & 3.987 & 5.014 & 4.439 & 4.208 & 3.812 \\
\hline 6 & Basin perimeter $(\mathrm{P})(\mathrm{km})$ & 86.710 & 62.717 & 59.179 & 57.898 & 45.548 & 51.266 \\
\hline 7 & Basin length $\left(\mathrm{L}_{\mathrm{b}}\right)$ & 25.419 & 23.256 & 20.965 & 18.964 & 15.900 & 15.309 \\
\hline 8 & Basin area $(\mathrm{A})\left(\mathrm{km}^{2}\right)$ & 222.756 & 172.291 & 114.271 & 188.000 & 97.193 & 98.410 \\
\hline 9 & Basin relief $\left(\mathrm{B}_{\mathrm{h}}\right)(\mathrm{m})$ & 825 & 846 & 786 & 730 & 560 & 421 \\
\hline 10 & Relief ratio $\left(\mathrm{R}_{\mathrm{r}}\right)$ & 0.032 & 0.037 & 0.037 & 0.038 & 0.035 & 0.027 \\
\hline 11 & Elongation ratio $\left(\mathrm{R}_{\mathrm{e}}\right)$ & 0.662 & 0.636 & 0.575 & 0.815 & 0.699 & 0.730 \\
\hline 12 & Circularity ratio $\left(\mathrm{R}_{\mathrm{c}}\right)$ & 0.372 & 0.550 & 0.409 & 0.704 & 0.588 & 0.470 \\
\hline 13 & Lemniscate ratio (k) & 0.725 & 0.784 & 0.961 & 0.478 & 0.650 & 0.595 \\
\hline 14 & Drainage density $\left(D_{d}\right)\left(\mathrm{km} / \mathrm{km}^{2}\right)$ & 1.891 & 1.755 & 1.587 & 1.711 & 1.738 & 1.718 \\
\hline 15 & Stream frequency $\left(\mathrm{F}_{\mathrm{s}}\right)$ & 1.818 & 2.00 & 1.688 & 1.771 & 1.707 & 1.829 \\
\hline 16 & Form factor $\left(\mathrm{R}_{\mathrm{f}}\right)$ & 0.344 & 0.318 & 0.259 & 0.522 & 0.313 & 0.419 \\
\hline 17 & Shape factor $\left(B_{s}\right)$ & 2.900 & 3.139 & 3.846 & 1.912 & 2.601 & 2.381 \\
\hline 18 & Drainage texture (Dt) & 4.670 & 5.500 & 3.261 & 5.751 & 3.644 & 3.511 \\
\hline 19 & Dissection index $\left(\mathrm{D}_{\mathrm{Is}}\right)$ & 0.504 & 0.508 & 0.489 & 0.472 & 0.392 & 0.326 \\
\hline 20 & Ruggedness number $\left(\mathrm{R}_{\mathrm{n}}\right)$ & 1.560 & 1.484 & 1.247 & 1.249 & 0.973 & 0.723 \\
\hline 21 & Drainage intensity $\left(\mathrm{D}_{\mathrm{I}}\right)$ & 0.961 & 1.139 & 1.063 & 1.035 & 0.982 & 1.064 \\
\hline 22 & Length of over land flow $\left(\mathrm{L}_{0}\right) \mathrm{Km}$ & 0.945 & 0.877 & 0.793 & 0.855 & 0.869 & 0.859 \\
\hline 23 & Hypsometric integral $\left(\mathrm{H}_{\mathrm{i}}\right)^{*}$ & 0.720 & 0.700 & 0.740 & 0.800 & 0.800 & 0.77 \\
\hline
\end{tabular}

\footnotetext{
"Source: [39].
} 
Table 3. Morphometric characteristics of dip slope watersheds.

\begin{tabular}{|c|c|c|c|c|c|c|c|}
\hline Par. No. & Morphometric parameters & W.Wuheida & W.Huseinan & $\begin{array}{l}\text { W.Tawayil } \\
\text { el Hamd }\end{array}$ & W.el Batra & $\begin{array}{l}\text { W.Aub } \\
\text { Tarfa } 1\end{array}$ & $\begin{array}{l}\text { W.Aub } \\
\text { Tarfa } 2\end{array}$ \\
\hline 1 & Stream order (u) & VI & IV & VI & $\mathrm{V}$ & VI & IV \\
\hline 2 & No. of streams $\left(\mathrm{N}_{\mathrm{u}}\right)$ (Total) & 356 & 239 & 722 & 412 & 786 & 189 \\
\hline 3 & Stream length $\left(\mathrm{L}_{\mathrm{u}}\right)$ (Total) Km & 402.657 & 246.086 & 863.549 & 455.759 & 881.136 & 204.957 \\
\hline 4 & Mean stream length $\left(\mathrm{L}_{\mathrm{sm}}\right)(\mathrm{km})$ & 1.131 & 1.029 & 1.196 & 1.106 & 1.121 & 1.084 \\
\hline 5 & Mean bifurcation ratio $\left(\mathrm{R}_{\mathrm{bm}}\right)$ & 4.212 & 4.825 & 4.595 & 4.573 & 5.010 & 5.388 \\
\hline 6 & Basin perimeter $(\mathrm{P})(\mathrm{km})$ & 73.981 & 99.998 & 106.900 & 108.828 & 99.539 & 73.857 \\
\hline 7 & Basin length $\left(L_{b}\right)$ & 23.815 & 42.193 & 41.609 & 35.353 & 37.984 & 30.084 \\
\hline 8 & Basin area $(\mathrm{A})\left(\mathrm{km}^{2}\right)$ & 235.583 & 141.246 & 466.985 & 257.098 & 491.850 & 118.284 \\
\hline 9 & Basin relief $\left(\mathrm{B}_{\mathrm{h}}\right)(\mathrm{m})$ & 514 & 714 & 682 & 673 & 532 & 388 \\
\hline 10 & Relief ratio $\left(\mathrm{R}_{\mathrm{r}}\right)$ & 0.021 & 0.016 & 0.016 & 0.019 & 0.014 & 0.012 \\
\hline 11 & Elongation ratio $\left(\mathrm{R}_{\mathrm{e}}\right)$ & 0.802 & 0.317 & 0.585 & 0.511 & 0.658 & 0.402 \\
\hline 12 & Circularity ratio $\left(\mathrm{R}_{\mathrm{c}}\right)$ & 0.540 & 0.177 & 0.513 & 0.272 & 0.623 & 0.272 \\
\hline 13 & Lemniscate ratio (k) & 0.494 & 3.150 & 0.926 & 1.215 & 0.773 & 1.960 \\
\hline 14 & Drainage density $\left(D_{d}\right)\left(\mathrm{km} / \mathrm{km}^{2}\right)$ & 1.709 & 1.742 & 1.849 & 1.772 & 1.791 & 1.732 \\
\hline 15 & Stream frequency $\left(F_{s}\right)$ & 1.509 & 1.692 & 1.546 & 1.602 & 1.598 & 1.597 \\
\hline 16 & Form factor $\left(\mathrm{R}_{\mathrm{f}}\right)$ & 0.364 & 0.079 & 0.269 & 0.205 & 0.340 & 0.127 \\
\hline 17 & Shape factor $\left(B_{s}\right)$ & 1.977 & 120.603 & 3.707 & 4.613 & 2.933 & 7.843 \\
\hline 18 & Drainage texture (Dt) & 4.812 & 2.930 & 6.753 & 3.785 & 7.896 & 2.558 \\
\hline 19 & Dissection index $\left(\mathrm{D}_{\mathrm{Is}}\right)$ & 0.297 & 0.427 & 0.424 & 0.411 & 0.370 & 0.286 \\
\hline 20 & Ruggedness number $\left(\mathrm{R}_{\mathrm{n}}\right)$ & 0.878 & 1.230 & 1.261 & 1.192 & 0.952 & 2.404 \\
\hline 21 & Drainage intensity $\left(D_{I}\right)$ & 0.882 & 0.971 & 0.836 & 0.904 & 0.892 & 0.922 \\
\hline 22 & Length of over land flow $\left(\mathrm{L}_{0}\right) \mathrm{Km}$ & 0.854 & 0.871 & 0.924 & 0.886 & 0.895 & 0.866 \\
\hline 23 & Hypsometric integral $\left(\mathrm{H}_{\mathrm{i}}\right)^{*}$ & 0.900 & 0.920 & 0.850 & 0.850 & 0.860 & 0.840 \\
\hline
\end{tabular}

*Source: [39].

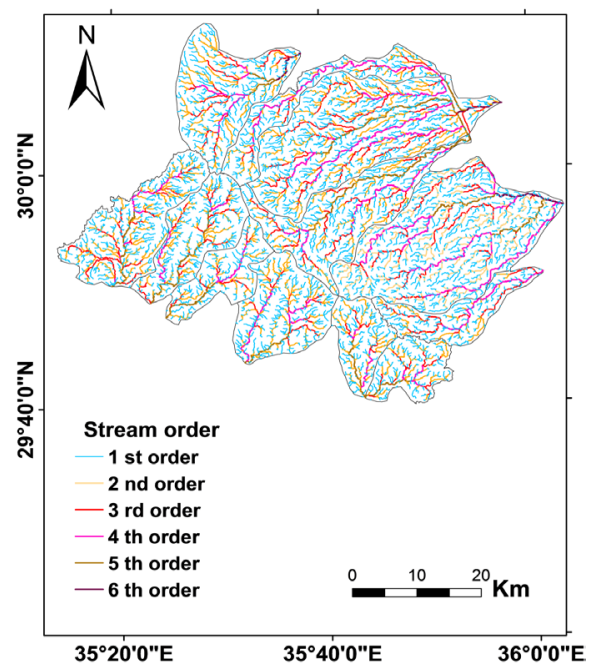

Figure 8. Stream order of the Ras En Naqb watersheds. 
Table 4. Morphometric characteristics of faulted-erosional slope watersheds.

\begin{tabular}{|c|c|c|c|c|c|c|}
\hline \multicolumn{7}{|c|}{ Wadi Jamam watershed } \\
\hline Par. No. & Morphometric parameters & & & tream ord & & \\
\hline 1 & Stream order (u) (5) & I & II & III & IV & $\mathrm{V}$ \\
\hline 2 & No. of streams $\left(\mathrm{N}_{\mathrm{u}}\right)$ (Total) (405) & 307 & 73 & 19 & 5 & 1 \\
\hline 3 & Stream length $\left(\mathrm{L}_{\mathrm{u}}\right)$ (Total) $(421.253 \mathrm{~km})$ & 200.120 & 123.388 & 62.573 & 18.140 & 17.032 \\
\hline 4 & Mean stream length $\left(\mathrm{L}_{\mathrm{sm}}\right)(1.040 \mathrm{~km})$ & 0.651 & 1.690 & 3.293 & 3.628 & 17.032 \\
\hline 5 & Stream length ratio $\left(\mathrm{R}_{\mathrm{L}}\right)$ & & $\begin{array}{c}0.616 \\
\text { II/I }\end{array}$ & $\begin{array}{c}0.507 \\
\text { III/II }\end{array}$ & $\begin{array}{l}0.289 \\
\text { IV/III }\end{array}$ & $\begin{array}{l}0.938 \\
\text { V/IV }\end{array}$ \\
\hline 6 & Bifurcation ratio( $\left.\mathrm{R}_{\mathrm{b}}\right)$ & & $\begin{array}{c}4.205 \\
\mathrm{I} / \mathrm{II}\end{array}$ & $\begin{array}{c}3.842 \\
\text { II/III }\end{array}$ & $\begin{array}{l}3.800 \\
\text { III/IV }\end{array}$ & $\begin{array}{c}5 \\
\text { IV/V }\end{array}$ \\
\hline \multicolumn{7}{|c|}{ Wadi Hanout watershed } \\
\hline Par. No. & Morphometric parameters & & & tream ord & & \\
\hline 1 & Stream order (u) (5) & I & II & III & IV & $\mathrm{V}$ \\
\hline 2 & No. of stream order $\left(\mathrm{N}_{\mathrm{u}}\right)$ (Total) (345) & 260 & 66 & 15 & 3 & 1 \\
\hline 3 & Stream length $\left(\mathrm{L}_{\mathrm{u}}\right)$ (Total) (302.434 km) & 148.428 & 72.734 & 39.358 & 17.312 & 24.602 \\
\hline 4 & Mean stream length $\left(\mathrm{L}_{\mathrm{sm}}\right)(0.876 \mathrm{~km})$ & 0.570 & 1.102 & 2.623 & 5.770 & 24.602 \\
\hline 5 & Stream length ratio $\left(\mathrm{R}_{\mathrm{L}}\right)$ & & $\begin{array}{c}0.490 \\
\text { II/I }\end{array}$ & $\begin{array}{c}0.541 \\
\text { III/II }\end{array}$ & $\begin{array}{l}0.439 \\
\text { IV/III }\end{array}$ & $\begin{array}{l}1.421 \\
\text { V/IV }\end{array}$ \\
\hline 6 & Bifurcation ratio $\left(\mathrm{R}_{\mathrm{b}}\right)$ & & $\begin{array}{c}3.939 \\
\text { I/II }\end{array}$ & $\begin{array}{c}4.400 \\
\text { II/III }\end{array}$ & $\begin{array}{c}5 \\
\text { III/IV }\end{array}$ & $\begin{array}{c}3 \\
\text { IV/V }\end{array}$ \\
\hline \multicolumn{7}{|c|}{ Wadi Jaded watershed } \\
\hline Par. No. & Morphometric parameters & & & tream ord & & \\
\hline 1 & Stream order (u) (5) & I & II & III & IV & $\mathrm{V}$ \\
\hline 2 & No. of streams $\left(\mathrm{N}_{\mathrm{u}}\right)$ (Total) (193) & 154 & 29 & 7 & 2 & 1 \\
\hline 3 & Stream length $\left(\mathrm{L}_{\mathrm{u}}\right)$ (Total) $(181.362 \mathrm{~km})$ & 91.476 & 46.734 & 17.944 & 11.970 & 13.238 \\
\hline 4 & Mean stream length $\left(\mathrm{L}_{\mathrm{sm}}\right)(0.939 \mathrm{~km})$ & 0.594 & 1.611 & 2.563 & 5.985 & 13.238 \\
\hline 5 & Stream length ratio $\left(\mathrm{R}_{\mathrm{L}}\right)$ & & $\begin{array}{c}0.510 \\
\text { II/I }\end{array}$ & $\begin{array}{c}0.383 \\
\text { III/II }\end{array}$ & $\begin{array}{c}0.667 \\
\text { IV/III }\end{array}$ & $\begin{array}{l}1.105 \\
\text { V/IV }\end{array}$ \\
\hline 6 & Bifurcation ratio $\left(\mathrm{R}_{\mathrm{b}}\right)$ & & $\begin{array}{c}5.310 \\
\mathrm{I} / \mathrm{II}\end{array}$ & $\begin{array}{c}4.142 \\
\text { II/III }\end{array}$ & $\begin{array}{l}3.500 \\
\text { III/IV }\end{array}$ & $\begin{array}{c}2 \\
\text { IV/V }\end{array}$ \\
\hline \multicolumn{7}{|c|}{ Wadi Ghafir watershed } \\
\hline Par. No. & Morphometric parameters & & & tream ord & & \\
\hline 1 & Stream order (u) (5) & I & II & III & IV & $\mathrm{V}$ \\
\hline 2 & No. of streams $\left(\mathrm{N}_{\mathrm{u}}\right)$ (Total) (333) & 258 & 59 & 12 & 3 & 1 \\
\hline 3 & Stream length $\left(\mathrm{L}_{\mathrm{u}}\right)$ (Total) (321.742 km) & 166.076 & 87.640 & 29.834 & 25.548 & 12.644 \\
\hline 4 & Mean stream length $\left(\mathrm{L}_{\mathrm{sm}}\right)(0.966 \mathrm{~km})$ & 0.643 & 1.485 & 2.486 & 8.516 & 12.644 \\
\hline 5 & Stream length ratio $\left(\mathrm{R}_{\mathrm{L}}\right)$ & & $\begin{array}{c}0.527 \\
\text { II/I }\end{array}$ & $\begin{array}{c}0.340 \\
\text { III/II }\end{array}$ & $\begin{array}{c}0.856 \\
\text { IV/III }\end{array}$ & $\begin{array}{l}0.494 \\
\text { V/IV }\end{array}$ \\
\hline 6 & Bifurcation ratio $\left(\mathrm{R}_{\mathrm{b}}\right)$ & & $\begin{array}{c}4.372 \\
\text { I/II }\end{array}$ & $\begin{array}{c}4.916 \\
\text { II/III }\end{array}$ & $\begin{array}{c}4 \\
\text { III/IV }\end{array}$ & $\begin{array}{c}3 \\
\mathrm{IV} / \mathrm{V}\end{array}$ \\
\hline
\end{tabular}




\section{Continued}

\begin{tabular}{|c|c|c|c|c|c|c|}
\hline \multicolumn{7}{|c|}{ Wadi Hafir watershed } \\
\hline Par. No. & Morphometric parameters & & & ream ord & & \\
\hline 1 & Stream order (u) (5) & I & II & III & IV & $\mathrm{V}$ \\
\hline 2 & No. of streams $\left(\mathrm{N}_{\mathrm{u}}\right)$ (Total) $(166)$ & 125 & 31 & 6 & 3 & 1 \\
\hline 3 & Stream length $\left(\mathrm{L}_{\mathrm{u}}\right)$ (Total) $(168.939 \mathrm{~km})$ & 80.343 & 51.763 & 13.563 & 11.123 & 12.147 \\
\hline 4 & Mean stream length $\left(\mathrm{L}_{\mathrm{sm}}\right)$ (1.017 km) & 0.642 & 1.669 & 2.260 & 3.707 & 12.147 \\
\hline 5 & Stream length ratio $\left(\mathrm{R}_{\mathrm{L}}\right)$ & & $\begin{array}{c}0.644 \\
\text { II/I }\end{array}$ & $\begin{array}{c}0.262 \\
\text { III/II }\end{array}$ & $\begin{array}{l}0.820 \\
\text { IV/III }\end{array}$ & $\begin{array}{c}0.1 .092 \\
\text { V/IV }\end{array}$ \\
\hline 6 & Bifurcation ratio $\left(\mathrm{R}_{\mathrm{b}}\right)$ & & $\begin{array}{c}4.032 \\
\mathrm{I} / \mathrm{II}\end{array}$ & $\begin{array}{c}5.166 \\
\text { II/III }\end{array}$ & $\begin{array}{c}2 \\
\text { III/IV }\end{array}$ & $\begin{array}{c}3 \\
\text { IV/V }\end{array}$ \\
\hline \multicolumn{7}{|c|}{ Wadi Rabigh watershed } \\
\hline Par. No. & Morphometric parameters & & & ream ord & & \\
\hline 1 & Stream order (u) (5) & I & II & III & IV & $\mathrm{V}$ \\
\hline 2 & No. of streams $\left(\mathrm{N}_{\mathrm{u}}\right)$ (Total) (180) & 133 & 34 & 9 & 3 & 1 \\
\hline 3 & Stream length $\left(\mathrm{L}_{\mathrm{u}}\right)$ (Total) $(169.140 \mathrm{~km})$ & 80.446 & 48.200 & 21.998 & 8.611 & 9.885 \\
\hline 4 & Mean stream length $\left(\mathrm{L}_{\mathrm{sm}}\right)(0.939 \mathrm{~km})$ & 0.604 & 1.417 & 2.444 & 2.870 & 9.885 \\
\hline 5 & Stream length ratio $\left(\mathrm{R}_{\mathrm{L}}\right)$ & & $\begin{array}{c}0.599 \\
\text { II/I }\end{array}$ & $\begin{array}{c}0.456 \\
\text { III/II }\end{array}$ & $\begin{array}{l}0.391 \\
\text { IV/III }\end{array}$ & $\begin{array}{c}1.147 \\
\mathrm{~V} / \mathrm{IV}\end{array}$ \\
\hline 6 & Bifurcation ratio $\left(\mathrm{R}_{\mathrm{b}}\right)$ & & $\begin{array}{c}3.911 \\
\text { I/II }\end{array}$ & $\begin{array}{c}3.777 \\
\text { II/III }\end{array}$ & $\begin{array}{c}3 \\
\text { III/IV }\end{array}$ & $\begin{array}{c}3 \\
\mathrm{IV} / \mathrm{V}\end{array}$ \\
\hline
\end{tabular}

Table 5. Morphometric characteristics of dip slope watersheds.

\begin{tabular}{|c|c|c|c|c|c|c|c|}
\hline \multicolumn{8}{|c|}{ Wadi Wuheida watershed } \\
\hline Par. No. & Morphometric parameters & \multicolumn{6}{|c|}{ Stream order } \\
\hline 1 & Stream order $(\mathrm{u})(6)$ & I & II & III & IV & $\mathrm{V}$ & VI \\
\hline 2 & No. of streams $\left(\mathrm{N}_{\mathrm{u}}\right)$ (Total) (356) & 271 & 63 & 15 & 4 & 2 & 1 \\
\hline 3 & Stream length $\left(\mathrm{L}_{\mathrm{u}}\right)$ (Total) $(402.657 \mathrm{~km})$ & 200.860 & 104.462 & 55.948 & 23.369 & 10.835 & 7.183 \\
\hline 4 & Mean stream length $\left(\mathrm{L}_{\mathrm{sm}}\right)(1.131 \mathrm{~km})$ & 0.741 & 1.658 & 3.729 & 5.842 & 5.417 & 7.183 \\
\hline 5 & Stream length ratio $\left(\mathrm{R}_{\mathrm{L}}\right)$ & & $\begin{array}{c}0.520 \\
\text { II/I }\end{array}$ & $\begin{array}{c}0.535 \\
\text { III/II }\end{array}$ & $\begin{array}{c}0.417 \\
\text { IV/III }\end{array}$ & $\begin{array}{c}0.463 \\
\mathrm{~V} / \mathrm{IV}\end{array}$ & $\begin{array}{l}0.662 \\
\mathrm{VI} / \mathrm{V}\end{array}$ \\
\hline 6 & Bifurcation ratio $\left(\mathrm{R}_{\mathrm{b}}\right)$ & & $\begin{array}{c}4.301 \\
\text { I/II }\end{array}$ & $\begin{array}{c}4.200 \\
\text { II/III }\end{array}$ & $\begin{array}{l}3.750 \\
\text { III/IV }\end{array}$ & $\begin{array}{c}2 \\
\text { IV/V }\end{array}$ & $\begin{array}{c}2 \\
\mathrm{~V} / \mathrm{Vi}\end{array}$ \\
\hline \multicolumn{8}{|c|}{ Wadi Huseinan watershed } \\
\hline Par. No. & Morphometric parameters & \multicolumn{6}{|c|}{ Stream order } \\
\hline 1 & Stream order (u) (4) & I & & II & III & & IV \\
\hline 2 & No. of streams $\left(\mathrm{N}_{\mathrm{u}}\right)$ (Total) (239) & 189 & & 40 & 9 & & 1 \\
\hline 3 & Stream length $\left(\mathrm{L}_{\mathrm{u}}\right)$ (Total) $(246.086 \mathrm{~km})$ & 121.183 & & 52.140 & 25.837 & & 46.926 \\
\hline 4 & Mean stream length $\left(\mathrm{L}_{\mathrm{sm}}\right)(1.029 \mathrm{~km})$ & 0.641 & & 1.303 & 1.033 & & 46.926 \\
\hline 5 & Stream length ratio $\left(\mathrm{R}_{\mathrm{L}}\right)$ & & & $\begin{array}{c}0.430 \\
\mathrm{II} / \mathrm{I}\end{array}$ & $\begin{array}{c}0.495 \\
\text { III/II }\end{array}$ & & $\begin{array}{l}1.816 \\
\text { IV/III }\end{array}$ \\
\hline 6 & Bifurcation ratio $\left(\mathrm{R}_{\mathrm{b}}\right)$ & & & $\begin{array}{c}4.725 \\
\text { I/II }\end{array}$ & $\begin{array}{c}4.444 \\
\text { II/III }\end{array}$ & & $\begin{array}{c}9 \\
\text { III/IV }\end{array}$ \\
\hline
\end{tabular}




\section{Continued}

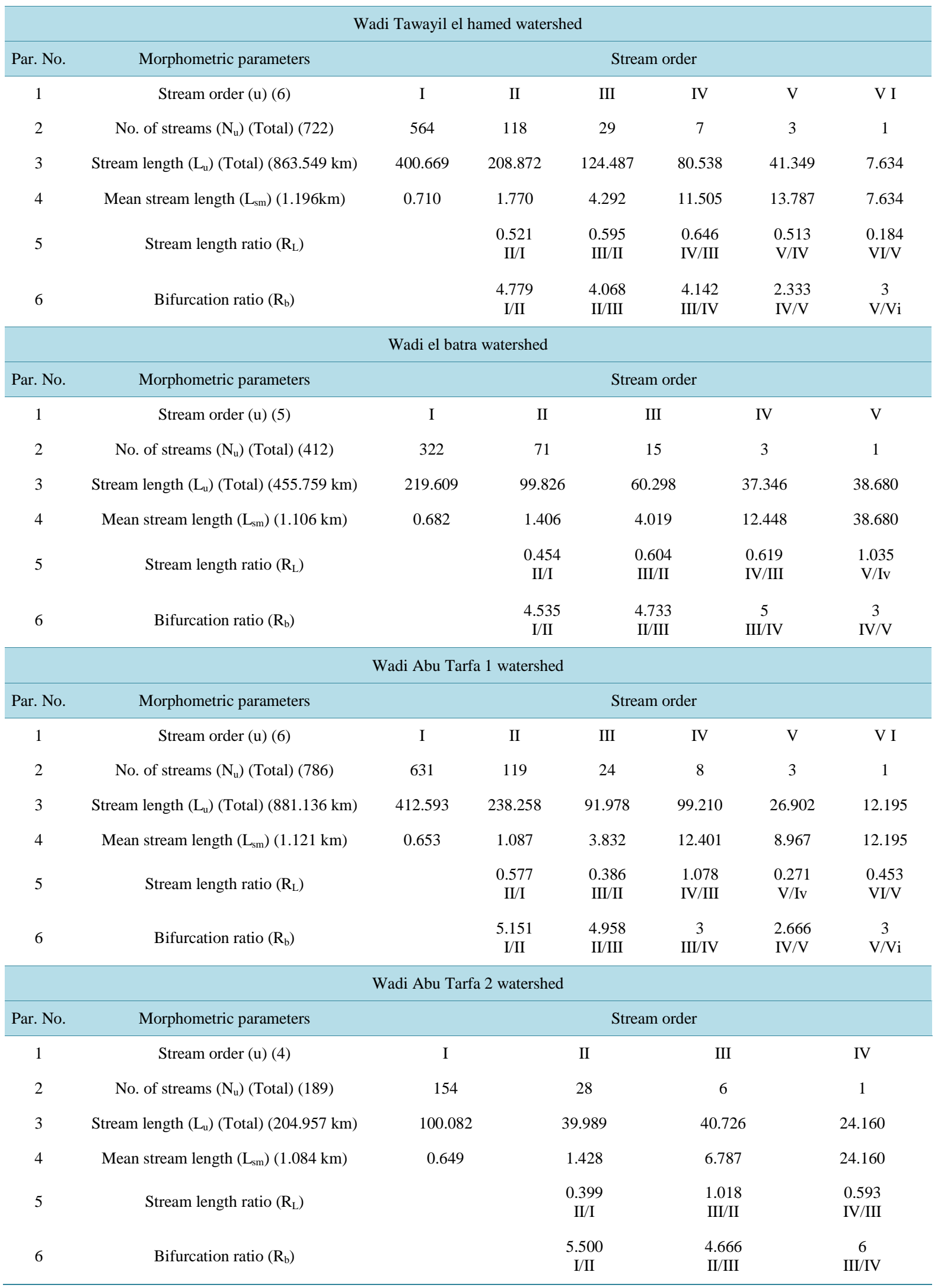




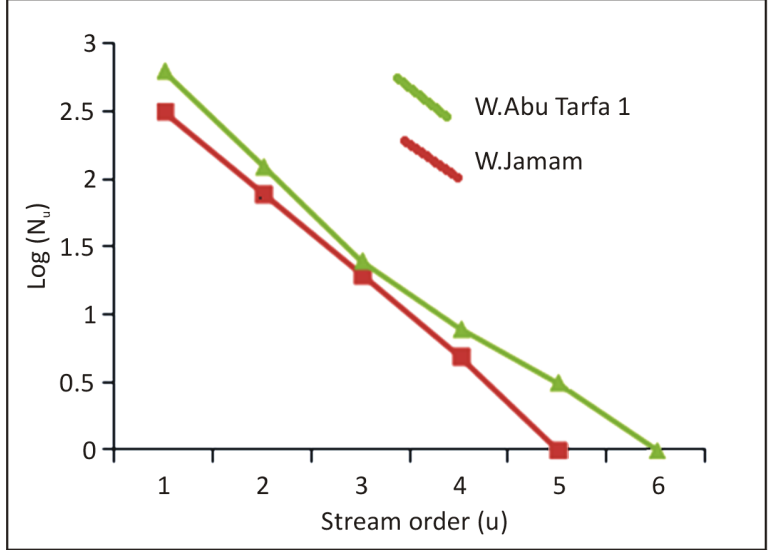

(a)

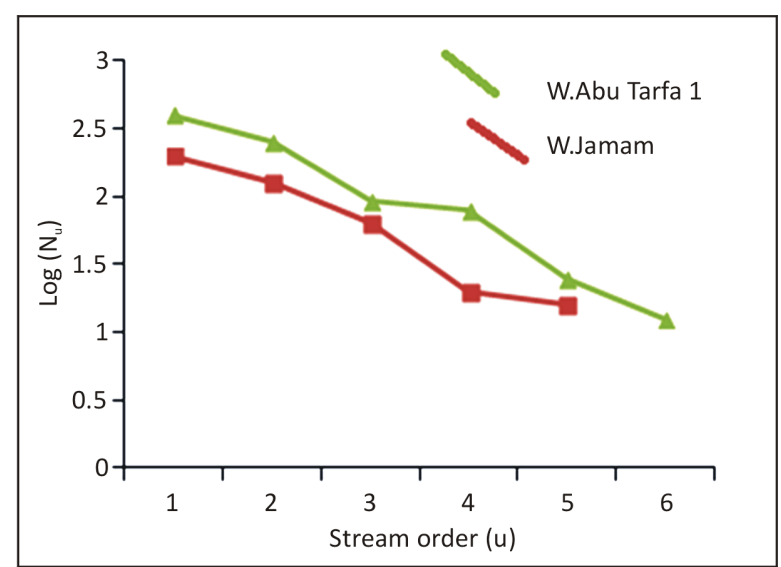

(b)

Figure 9. Horton's first law (a) and Horton’s second law (b) using W.Abu Tarfa 1 and W.Jamam watersheds.

streams constitute $75.7 \%$ and $78 \%$ of the total stream length related to faulted-erosional slope watersheds and dip slope catchments respectively. The stream length characteristics of all basins verify Horton's second law [7], the "law of stream length", which indicates that the average length of streams of each of the different orders in a drainage basin tends to closely approximate a direct geometric ratio. The geometric linear relationship is shown graphically when log values of these parameters are plotted on an ordinary graph (Figure 9(b)). However, slight deviations from a straight line are obvious at high and low orders due to uplifting of the Ras En Naqb escarpment and lowering of El-Jafr synsedimentary basin [23] [26].

2) Mean stream length $\left(L_{s m}\right)$ values for the faulted-erosional slope catchments vary from 0.837 to 6.9 , while $\mathrm{L}_{\mathrm{sm}}$ for the dip slope basins range from 0.87 to 6.01 .

3) Stream length ratio $\left(R_{L}\right)$ is the ratio between the mean length of streams of a given order to the mean length of streams in the next lower order. $\mathrm{R}_{\mathrm{L}}$ is considered a significant factor in relation to both drainage composition and geometric development of drainage basins [7]. A noticeable variation occurs in $\mathrm{R}_{\mathrm{L}}$ values between the streams of different orders pertaining to the faulted-erosional slope catchments $(0.494-1.421)$, and watersheds belong to the dip slopes $(0.184-1.816)$. This variation is attributed to morphological changes in slope and relief along both dip slopes and the faulted-erosional slopes, and the youth-age stage of geomorphic development of the watersheds as verified later through hypsometric analysis (Table 2 and Table 3).

4) Bifurcation ratio $\left(\mathrm{R}_{\mathrm{b}}\right)$ is elaborated by Horton [7] as an index of relief and dissection. Its value is about 2 for flat or rolling drainage basins, and up to 3 or 4 for mountainous or highly dissected drainage basins. Characteristically, $R_{b}$ values range between 3.66 and 6 for watersheds in which the geological structures distort the drainage pattern. By contrast, lower values of $R_{b}$ are representative for structurally less disturbed catchments without any distortion in drainage pattern [8] [9]. Abnormally high bifurcation ratio might be expected in regions of steeply dipping rock strata, where narrow strike valleys are confined between hogback ridges. The mean bifurcation ratios $\left(\mathrm{R}_{\mathrm{bm}}\right)$ for the dip slope catchments vary between 4.212 and 5.39. By contrast, the $\mathrm{R}_{\mathrm{bm}}$ values for the faulted-erosional slope basins vary between 3.75 - 5.014. Such high figures denote that drainage development of the watersheds is remarkably influenced by structural disturbances such as faulting, uplifting of the Ras En Naqb escarpment, subsidence of El Jafr basin, and rejuvenation of the drainage network.

\subsubsection{Basin Geometry}

A prominent variation exists in the values of morphometric parameters which represent basins geometry (basin area, basin length and basin perimeter). The areas of the dip slope catchments are varied. It ranges from 491.85 $\mathrm{km}^{2}$ (W.Abu Tarfa 1) to $118.28 \mathrm{~km}^{2}$ (W.Abu Tarfa 2), whereas the areas of faulted-erosional watersheds range from $97.193 \mathrm{~km}^{2}$ (W.Hafir) to $222.76 \mathrm{~km}^{2}$ (W.Jamam). Basin length of the faulted-erosional slope catchments ranges from $15.31 \mathrm{~km}$ (W.Rabigh) to $25.42 \mathrm{~km}$ (W.Jamam), while the basin length of the dip slope catchments varies between $23.82 \mathrm{~km}$ (W.Wuheida) and $42.19 \mathrm{~km}$ (W.Huseinan) (Table 2 and Table 3). Moreover, the perimeters of the faulted-erosional slope basins range between $45.55 \mathrm{~km}$ (W.Hafir) and $86.7 \mathrm{~km}$ (W.Jamam). Similarly, the perimeters of the dip slope watersheds are larger and range between $73.86 \mathrm{~km}$ (W.Abu Tarfa 2) and 
$108.83 \mathrm{~km}$ (W.el Batra). It is obvious that the values of the three geometric parameters characterizing the dip slope catchment are considerably higher than resembling parameters recognized for the faulted-erosional slope watersheds. Here, the dip slope catchments between the crest line of the escarpment and the base level of the wadis (El Jafr basin) are longer compared to those developed on the faulted-erosional slopes. Rejuvenation also resulted in wider, longer and larger watersheds.

1) Form factor $\left(R_{f}\right)$ is expressed as the ratio between the area of the catchment (A) and the square of the catchment length [7]. $\mathrm{R}_{\mathrm{f}}$ parameter has been developed to predict the intensity of a basin of a defined area. For a perfectly circular basin, the value of the form factor should always be less than 0.79 [40]. The smaller the value of $R_{f}(<0.45)$, the more the basin will be elongated. Catchments with high $R_{f}$ have peak flows of shorter duration, whereas elongated watersheds with low form factors have lower peak flow of longer duration [41] [42]. The watersheds of the dip slopes show a lower form factor, and vary from 0.14 to 0.47 . $\mathrm{R}_{\mathrm{f}}$ values related to four catchments are less than 0.56, while the values for two catchments are around 0.45 , indicating elongated shape and suggesting a flat hydrograph peak for longer duration. Flood flows of such elongated basins are easier to manage than watersheds developed towards rectangular to circular shape such as W.Rabigh $\left(\mathrm{R}_{\mathrm{f}}=0.68\right)$ and W.Ghafir $\left(\mathrm{R}_{\mathrm{f}}\right.$ $=0.522$ ) belonging to the faulted-erosional slope of Ras En Naqb escarpment. Thus, high peak flows of shorter duration are expected during flash floods [43] [44]. This is verified by previous floods recorded in the AqabaMa'an-Ras En Naqb area [32] [33] [45] [46]. Therefore, the morphological characteristics of a watershed have powerful impacts on watershed hydrology.

2) Elongation ratio $\left(R_{e}\right)$ is defined as the ratio between the diameter of the circle of the area as represented by the drainage basin to the maximum basin length [8]. Strahler [3] stated that $R_{e}$ values vary generally between 0.6 to 1.0 over a wide range of climate and geological conditions. Values close to 1.0 are characteristic of regions with very low relief, whereas values in the range of 0.6 - 0.8 are normally diagnostic of watersheds with high relief and steep slopes. Where $R_{e}$ approaches 1.0, the shape of the drainage basin approaches a circle [8]. It has been concluded that a circular basin is more efficient in runoff than is an elongated one [47]. $R_{e}$ values for W.Rabigh and W.Ghafir which belong to the faulted-erosional slopes are greater than 0.7 ( 0.73 and 0.815 respectively) (Table 2 and Table 3), while $\mathrm{R}_{\mathrm{e}}$ values for the other four wadis are less than 0.70 . Such figures indicate that W.Rabigh and W.Ghafir are approaching the circular shape, whereas the other four wadis (Table 3) are elongated or less elongated with high relief and steep slopes. The circular watersheds have a quick; although lower; hydrograph peak compared to an oval watershed (i.e. W.Ghafir), or a less elongated and elongated watershed. This implies that the other four catchments show shorter time to peak.

3) Shape factor $\left(B_{s}\right)$ is calculated by dividing the square of the length of a basin by the area of the basin [7], and is considered in inverse proportion to the form factor $\left(\mathrm{R}_{\mathrm{f}}\right)$. The shape of the drainage basin along with the length and relief affect the rate of water and sediment yield. $\mathrm{B}_{\mathrm{s}}$ values for the catchments of the dip slope range from 1.977 to 12.6 with an average of 5.596; thus, it is expected to have the shorter basin lag time, whereas; the Bs values for the watersheds of the faulted-erosional slopes vary from 1.9 to 3.85 with an average of 2.76. Therefore, it may have a longer basin lag time.

4) Lemniscate ratio $(\mathrm{k})$ is a measure elaborated to describe how closely the actual drainage basin shape approaches the loop of a lemniscates [48] [49]. It has been reported that for describing the drainage basin shape accurately, it is necessary to determine the lemniscate shape which the basin most nearly approaches. In this context, the lemniscate ratio allows for distinguishing regional variation of drainage basin shapes. Therefore, it is considered a useful index to differentiate one morphometric region from another, and to express quantitatively the structural control over basin shape; as, for example; in the effect of varying angles of dip on the shapes of drainage basins developed on the dip slopes. The $\mathrm{k}$ values for the dip slope catchment range from 0.494 to 3.15 with an average of 1.42 , whereas the $\mathrm{k}$ values for the watersheds of the faulted-erosional slopes vary from 0.478 to 0.961 with an average of 0.698 . This indicates that dip slope catchments are mostly elongated in shape, and have delayed time to peak flow. By contrast, the faulted-erosional slope watersheds are less elongated and have a shorter time to peak flow.

5) Circularity ratio $\left(R_{c}\right)$ refers to the ratio of catchment area $(A)$ to the area of circle having the same circumference as the perimeter of the catchment [50]. $R_{c}$ is controlled by the length and frequency of the streams, geological structures, landuse, land cover, climate, relief and slope steepness of the catchment. Drainage basins with a range of circularity ratios of 0.4 to 0.5 were described by Miller [50], indicating that they are strongly elongated, highly permeable, with homogeneous geological materials. $\mathrm{R}_{c}$ for the watersheds developed on the faulted-erosional slope is in the range from 0.45 to 0.85 indicating that these watersheds are characterized by 
high relief, elongated and relatively permeable surface resulting in greater basin lag times, while catchments belonging to the dip slopes show delayed time to peak flow, and most wadis of the faulted-erosional slopes show shorter time to peak. It can be concluded that $R_{f}, R_{e}$ and $R_{c}$ significantly influence the hydrological response of the Ras En Naqb watersheds. Also, the combination with basin shape and the arrangement of stream segments has a direct influence on the size and shape of flood peak [51], which is indicative for mountainous arid catchments of Jordan.

6) Drainage texture $\left(D_{t}\right)$ denotes relative spacing of drainage lines in a fluvially dissected terrain. It is defined as the total number of stream segments of all orders per perimeter of the drainage basin [7]. $D_{t}$ represents one of the main concepts in drainage basin geomorphology. $\mathrm{D}_{\mathrm{t}}$ is influenced by several intrinsic physical factors such as: climate, rainfall, vegetation, soils, lithology, infiltration-capacity, relief and stage of basin development. Smith [52] has identified five different texture categories: drainage density $<2$ indicates very coarse texture, between 2 and 4 is described as coarse texture, between 4 and 6 is moderate, between 6 and 8 is fine, and $>8$ is very fine drainage texture. $D_{t}$ values for three dip slope catchments are less than 2 and the three watersheds are greater than 3 , but less than 4 , whereas; $D_{t}$ values for two catchments of the faulted-erosional slopes are $<2$, and four catchments have $D_{t}$ values between 2 and 4. Thus, the Ras En Naqb watersheds exhibit a very coarse to coarse drainage texture, which indicates the presence of relatively resistant, permeable materials with moderate relief.

\subsubsection{Drainage Texture Parameters}

1) Stream frequency $\left(F_{s}\right)$ represents the ratio of the total number of streams $\left(N_{u}\right)$ in a basin to the basin area (A), and is defined as the number of streams per unit of area [7]. Generally, the value of stream frequency ranges from 3.91 to 9.99. $\mathrm{F}_{\mathrm{s}}$ values depend on lithology of the catchment, and reflect the texture of the drainage network. The Fs values are positively correlated with $D_{d}$ values of a watershed, which means that the increase in stream population is connected to that of drainage density [43]. High stream frequency means more percolation with respect to drainage density and thus more groundwater potential [20]. The observed stream frequency $\left(\mathrm{F}_{\mathrm{s}}\right)$ values range from 1.688 to 2.00 for the faulted-erosional slope catchments, and from 1.509 to 1.692 for dip slope watersheds. It is obvious that $\mathrm{F}_{\mathrm{s}}$ values indicate steep slopes, with low permeability rocks, thus facilitating less infiltration and greater surface flow and high flooding potential [53].

2) Drainage density $\left(D_{d}\right)$ is defined as the closeness of spacing of channels, and considered a quantitative expression of terrain dissection and runoff potential of the catchment. Drainage density is a measure of the total lengths of streams in a catchment per unit area. High drainage density of an area implies high runoff, consequently low infiltration rate, whereas; low drainage density of an area implies high runoff, and consequently low drainage density of an area refers to low runoff and high infiltration [21]. Other significant parameters determining $D_{d}$ are infiltration-capacity of the soils, and initial resistance of terrain to erosion. The poorly drained basins have a drainage density of 2.74, while the well-drained one has a density of 0.73 or one-forth as great [7]. The $D_{d}$ values for Ras En Naqb catchments vary from 1.6 - 1.9 for the faulted-erosional slope to 1.7 - 1.85 for the dip slope catchments. Relatively high $D_{d}$ values for the faulted-erosional slopes compared to the dip slope catchments are indicative of the presence of rugged terrain especially in the middle catchments. Also, the persistence of steep slopes $\left(25^{\circ}-45^{\circ}\right.$, and $60^{\circ}$, denotes high runoff and low infiltration-capacity. However, the lower catchments of the faulted-erosional scarp belong to the inselberg landscape (of southern Jordan), and the upper catchments exhibit coarse texture terrain. Therefore, the presence of headward-eroding streams, and fault controlled canyons in the middle catchments are probably features inherited from pluvials recognized in the Mediterranean zone [28] [54].

3) Length of overland flow $\left(\mathrm{L}_{\mathrm{O}}\right)$ relates to the length of water over the ground before it becomes concentrated into definite steam channels. It is considered the most crucial independent variable affecting hydrological and geomorphological development of drainage basins. According to Horton [7] the average length of overland flow is relatively half the average distance between stream channels, and thus, is approximately equal to half of drainage density. Length of overland flow relates inversely to the average channel slope [15]. The $\mathrm{L}_{0}$ values for the dip slope catchments range from 0.584 to 0.921 , with an average of 0.882 , whereas $L_{o}$ values for the faultederosional slopes vary from 0.855 to 0.945 , with an average of 0.866 , indicating very steep slopes and shorter flow paths on the faulted-erosional slopes, and relatively moderate-steep slopes and longer flow paths characterizing the dip slope catchments.

4) Drainage intensity $\left(D_{i}\right)$ is defined as the ratio of the stream frequency $\left(F_{s}\right)$ to the drainage density $\left(D_{d}\right)[55]$. 
Low values of drainage intensity indicates that stream frequency and drainage density have little influence on the degree to which the land surface of a basin has been lowered by denudational processes. The drainage intensity for the faulted-erosional watersheds varies from 0.961 to 1.139 , with an average of 1.04 while Di values for the dip slope catchments range from 0.836 to 0.971 , with an average of 0.901 . The $D_{i}$ values of the dip slope catchments are lower than those representing the faulted-erosional slope watersheds. This denotes that the latest watersheds are more susceptible to flooding, gullying and sliding. Repetitive inundations were recorded in Qa El Jafr to the northeast, and three other playas in the southwest.

\subsubsection{Relief Characteristics}

1) Basin relief $\left(B_{h}\right)$ or "total relief" of a watershed is defined as the difference in elevation between the highest and lowest points on the basin [8]. Commonly, relief measures are indicative of the potential energy of a drainage system present by virtue of elevation above a given datum [3]. Basin relief is a significant factor in understanding the denudational properties of the catchment, landforms and drainage networks evolution, overland flow, through flow, and erosional behavior of the terrain. The total relief of the dip slope catchments varies from $388 \mathrm{~m}$ (W.Abu Tarfa 2) to $714 \mathrm{~m}$ (W.Huseinan), while $\mathrm{B}_{\mathrm{h}}$ values for the watersheds of the faulted-erosional slopes range from $421 \mathrm{~m}$ (W.Rabigh) to $846 \mathrm{~m}$ (W.Hanout). High $B_{h}$ values are restricted to the western catchments of the Ras En Naqb escarpment where elevations are maximum. By contrast, low $B_{h}$ values dominate the eastern part of the escarpment, where the morphology is remarkably subdued. High $B_{h}$ values for the faultederosional watersheds indicate a high potential erosional energy of the drainage system especially during flooding. As a result of the sinking base level of El Jafr depression, the dip slope catchments are relatively of high potential energy during intense rainstorms.

2) Relief ratio $\left(R_{r}\right)$ is considered a reasonable mean to measure the overall steepness of a drainage basin. Also, it is an indicator of the intensity of erosion processes operating on the watershed slopes [40]. $R_{r}$ is defined as the ratio between the total relief (or basin relief $B_{h}$ ) of a catchment and the longest basin length parallel to the principal drainage line. $\mathrm{R}_{\mathrm{r}}$ normally increases with decreasing drainage area and size of a given catchment. Schumm [8] reported a close correlation between sediment loss per unit area and relief ratio. Relief ratio allows comparison of the relative relief of any basin regardless of differences in scale of topography. The $R_{r}$ values for the dip slope catchment range from 0.012 to 0.019 , whereas $R_{r}$ values for the faulted-erosional slopes vary from 0.027 to 0.038 . Low values of $R_{r}$ normally indicate the predominance of slow erosion processes, as in the case of the inselbergs landscape in southern Jordan [30]. However, higher values of $\mathrm{R}_{\mathrm{r}}$ in the faulted-erosional slope catchments imply that these wadis are characterized by more intense erosion as compared with wadi of the dip slope. Hence, W.Ghafir is more susceptible to erosion, and W.Abu Tarfa 2 is the least among all watersheds of the Ras En Naqb area, if this parameter alone is considered for erosion intensity evaluation.

3) Ruggedness number $\left(R_{n}\right)$ is defined as the product of drainage density $\left(D_{d}\right)$ and basin relief $\left(B_{h}\right)$ divided by 1000 [2] [3]. Observed values of ruggedness number range from low (i.e. 0.06) for smooth and subdued morphology to over 1.0 for sharp morphology, and very high, extreme values $(>2)$ characterizing badlands topography. Watersheds having high $\mathrm{R}_{\mathrm{n}}$ values can be described by high susceptibility to soil erosion, landsliding, and a high response to an increase in peak discharge. In the present investigation the $R_{n}$ value is minimum in W.Rabigh $\left(\mathrm{R}_{\mathrm{n}}=0.723\right.$; faulted-erosional catchment $)$ and maximum in W.Abu Tarfa $2\left(\mathrm{R}_{\mathrm{n}}=2.404\right.$; dip slope catchment). This implies that W.Rabigh is the least susceptible to erosion, and W.Abu Tarfa 2 is the most susceptible to erosion among all watersheds of the Ras En Naqb escarpment.

4) Dissection index $\left(D_{i s}\right)$ has been elaborated to evaluate the degree of dissection or vertical erosion, and the stage of landform development in a given catchment [55]. $\mathrm{D}_{\mathrm{is}}$ is the ratio between the total relief (or relative relief) and absolute relief of the basin, which always ranges between 0.0 (complete absence of dissection and thus the dominance of flat topography) and 1 for infrequent cases such as vertical cliff topography at the sea shore, or vertical escarpment of hillslope. Extreme values of $D_{\text {is }}$ certainly exceed 1 such as Wadi Kerak and other rivers/ wadis draining to the rift region of Jordan as an example [56] [57]. The $\mathrm{D}_{\text {is }}$ values vary from a minimum 0.286 (W.Abu Tarfa 2; a dip slope catchment) to a maximum of 0.508 (W.Hanout; a faulted-erosional slope watershed). The spatial variation in dissection index $\left(\mathrm{D}_{\mathrm{is}}\right)$ refers to the presence of relatively dissected terrain at the western part of the escarpment, and the subdued terrain at the eastern part. Moreover, the average values of $D_{\text {is }}$ are 0.45 for the faulted-erosional slope catchments, and 0.37 for the dip slope basins, which clearly indicates that the faulted-erosional slopes are more dissected compared to the dip slopes of the escarpment.

5) Hypsometric curve and hypsometric integral were elaborated to understand the geomorphic evolution, type 
of erosion processes and relative age of landforms, along with influence of tectonic, lithology and climate on watersheds morphology. In this regard, hypsometric means the relative proportion of an area at different elevations within a watershed; therefore, it represents the distribution of area with respect to altitude [2] [8]. The hypsometric curve, or the area-elevation analysis (expressing how much land lies between two contour lines), and the hypsometric integral of the Ras En Naqb watersheds have been calculated and prepared [58]. Differences in the shape of the hypsometric curve (HC), and the hypsometric integral (Hi) value are attributed mainly to the degree of disequilibria in the balance of erosion and tectonic factors [2]. The hypsometric curve expresses the volume of rock mass in the watershed and amount of erosion that has taken place in that catchment against the remaining mass. Thus, the hypsometric integral is employed as an estimator of the erosion status of a watershed. Strahler [2] [3] [59] classified the basins according to their stages of geomorphic development (based on the shape of the hypsometric curve and the value of the hypsometric integral) into three categories, such as: youth stage (convex $\mathrm{HC}$ curve, $\mathrm{Hi} \geq 0.60$ ), where the watershed is highly susceptible to erosion; equilibrium or mature stage(S-shaped $\mathrm{HC}$ curve, $0.30 \leq \mathrm{Hi} \leq 0.60$ ), and peneplain (old) or monadnock stage (concave HC curve, $\mathrm{Hi} \leq 0.30$ ). Such classification also provides an indication of the erosion status of watersheds, and reflects the interaction between tectonics and erosion [60]-[62]. The hypsometric curves of the faulted-erosional slope and the dip slope watersheds are all convex upward curves, and the hypsometric integral values vary from 0.70 to 0.80 for the faulted-erosional slope watersheds, and from 0.84 to 0.92 for dip slope watersheds. The value of the hypsometric integral for W.Hanout (a faulted-erosional slope watershed), for example, is 0.70 (Figure 10(a)), whereas the value of the hypsometric integral for W.Huseinan (a dip slope catchment) is 0.92 (Figure 10(b)). This indicates that both wadis are at the youth-age stage of geomorphic evolution, and the faulted-erosional slope watersheds are relatively older than the dip slope catchments Moreover, W.Hanout has been subjected to tectonic disturbances and severe erosion; therefore, it is approaching the late youth stage of geomorphic development.

\subsubsection{Watersheds Morphometry and Flash Floods Potential}

Variations in morphometric and morphological characteristics of these watersheds have influenced the potential of flash floods occurrence. High intensity rainstorms are common in southern Jordan, and occasionally have resulted in severe flash floods [32] [33]. The high total relief, steep slopes, and the dominance of bare lands, enable the occurrence of destructive floods in the downstream of the dip slope catchments which influence Ma'an city and the El Jafr depression, while, the faulted-erosional slope wadis inundated the playas east of Quweira during flooding. During the storm of 11 March 1966 for example, the Ras En Naqb climate station recorded 71.2 $\mathrm{mm}$, Ma'an $39 \mathrm{~mm}$, the highlands over looking Ma'an from the west recorded $60 \mathrm{~mm}$ falls in $4 \mathrm{~h}$, with an intensity of $15 \mathrm{~mm} / \mathrm{h}$, while the mean annual rainfall of Ma'an is $44 \mathrm{~mm}$, and Ras En Naqb is $140 \mathrm{~mm}$. Therefore, destructive flash floods occurred along the dip slope wadis. The Wadi Wuheida flash flood (dip slope wadi) caused severe damages to Ma'an city and the Amman-Aqaba road. The flash flood of the 1966 storm is classified as a 50-year return period. Low-magnitude flash floods (5 - 10 year return period) occurred in 1991, 1993, 1994, 2006, 2010, 2012, 2013, 2014, and 2015, and resulted in great damage to the Aqaba area including the highways and roads network. Field investigations following flash floods show that the playas of the inselbergs
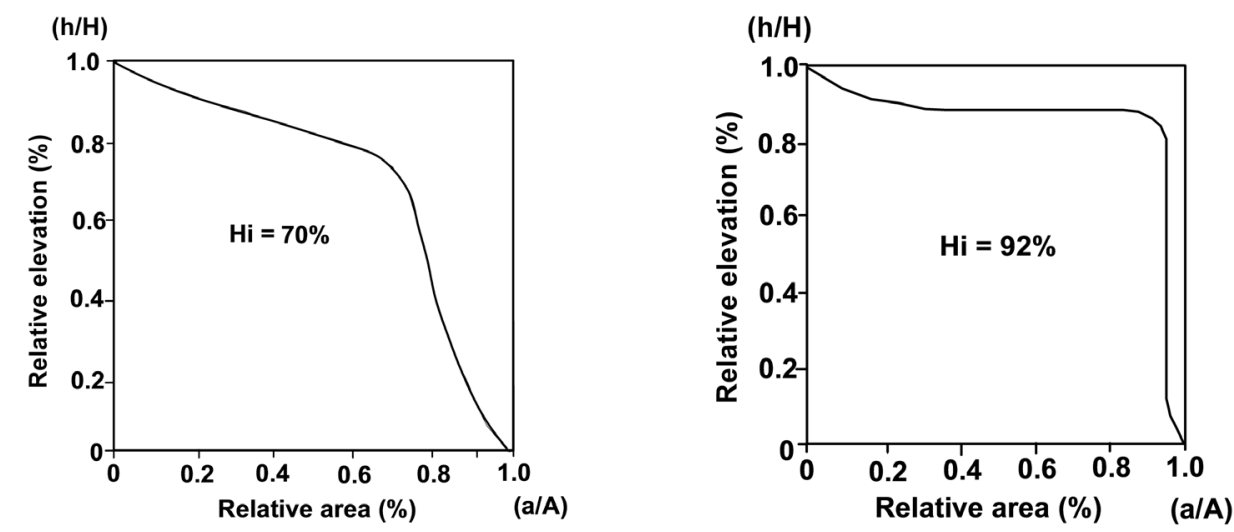

Figure 10. Hypsometric curves of W.Hanout (a) $(\mathrm{Hi}=70 \%)$ and W.Huseinan (b) $(\mathrm{Hi}=92 \%)$. 
landscape were flooded with water of $20 \mathrm{~cm}$ depth (Figure 11). Maximum intensity of flash floods occurred in the middle and lower watersheds of the wadis. Similarly, maximum inundation and standing flooding water achieved in the El-Jafr depression to the northeast, and the playas to the southwest of the Ras En Naqb escarpment.

The flooding risk for the twelve watersheds were determined using El-Shamy's diagrams as illustrated in Figure 12 and Figure 13. According to this approach, the relationship between bifurcation ratio $\left(\mathrm{R}_{\mathrm{b}}\right)$ and drainage density $\left(D_{d}\right)$, and then the relationship between $\left(R_{b}\right)$ and stream frequency $\left(F_{s}\right)$ have been utilized. The bifurcation ratios for the dip slope watersheds range between 4.2 and 5.4, with an average value of 4.72. By contrast, the $\mathrm{R}_{\mathrm{b}}$ values for the faulted-erosional slope catchments vary between 3.75 and 5.01, with an average value of 4.234, which indicates a noticeable control of geological structure on drainage network development. Furthermore, the calculated stream frequency $\left(\mathrm{F}_{\mathrm{s}}\right)$ values range from 1.688 to 2.0 for the faulted-erosional slope watersheds, and from 1.51 to 1.69 for dip slope basins. It is perceivable that $F_{s}$ values denote steep slopes, with low permeability rocks, thus facilitating less infiltration, greater runoff and high flooding potential. The watersheds exposed to flash floods were demarcated and assessed in order to determine catchments of low, intermediate and high flooding potential based on the relationship of two morphometric parameters $\left(R_{b} v s . D_{d}\right.$ and $R_{b} v s$. $\mathrm{F}_{\mathrm{s}}$ ), then the final flood hazard maps were generated with the aid of Arc GIS tool. Based on the relationship between $\mathrm{Rb}$ and Dd, watersheds nos. 1 (W.Rabigh) and 2 (W.Hafir) of the faulted-erosional slopes are categorized as of high susceptibility to flooding (Figure 12). Whereas, watersheds nos. 3 (W.Ghafir), 4 (W.Jaded), 5 (W.Hanout), and 6 (W.Jamam) represent the category of intermediate flooding susceptibility. By contrast, watersheds nos. 1 (W.Abu Tarfa 2) and 4 (W.Tawayil el Hamd) of the dip slopes are classified as of high flooding potential (Figure 12). Similarly, catchments no. 3 (W.el Batra) and 6 (W.Wuheida) are characterized as of intermediate susceptibility to flooding (Figure 12). Furthermore, watersheds nos. 2 (W.Abu Tarfa 1) and 5 (W.Huseinan) are considered of low susceptibility to flooding. More consistent results of floods hazard susceptibility exist in El-Shamey's approach for watersheds categorized based on the relationship between Rb vs. Fs. In this context, Figure 13 shows that all the six faulted-erosional slope watersheds are classified as catchments of high flooding susceptibility. By contrast, four dip slope catchments are categorized as of intermediate flooding liability. (W.Abu Tarfa 2 1; W.el Batra 3; W.Tawayil el Hamd 4; and W.Wuheida 6). Nevertheless, W.Abu Tarfa 1 and W.Huseinan are ranked under low flooding susceptibility. It can be concluded that ten watersheds (83.3\%) are classified under high and intermediate flooding susceptibility (Figure 12 and Figure 13). The faulted-erosional slope watersheds are generally more hazardous in terms of flooding. Thus, the protection of Ma'an city (located on W.Wuheida), El Jafr rural Bedouin settlement, and Amman-Aqaba highway from repetitive flooding is essential to maintain sustainable development across the Ras En Naqb-Ma'an area in the future.

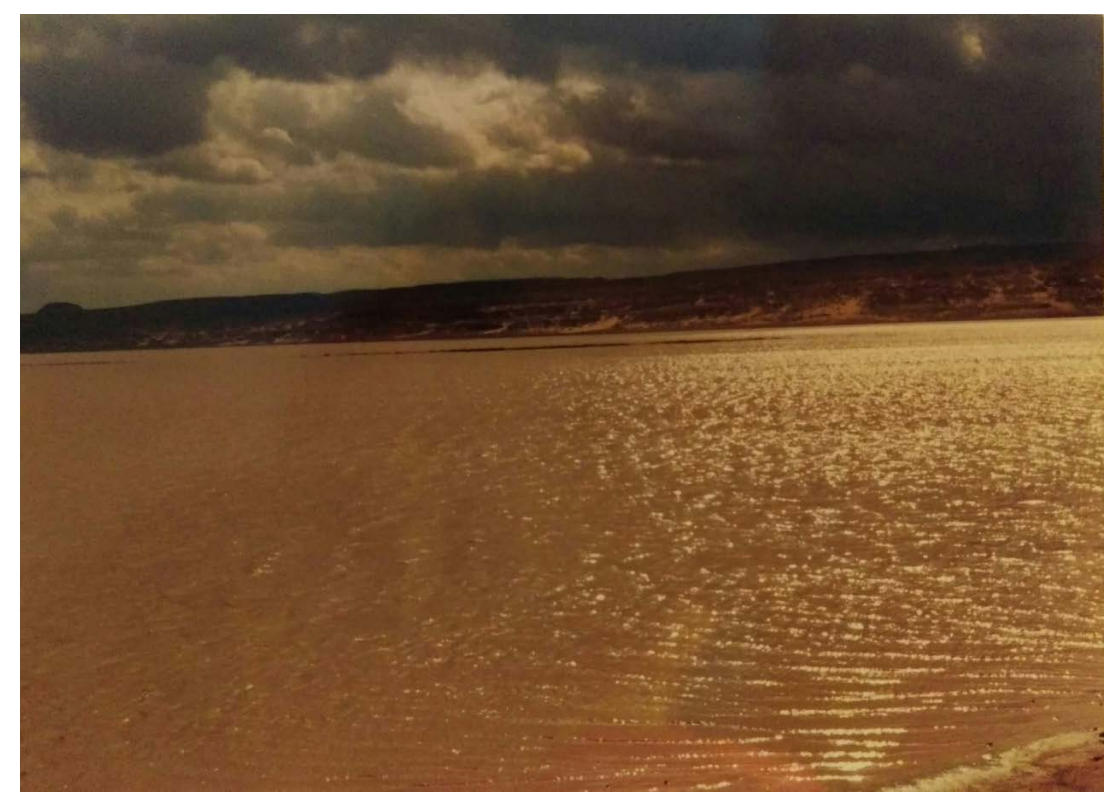

Figure 11. Inundation of playa due to flooding (March 1994). 


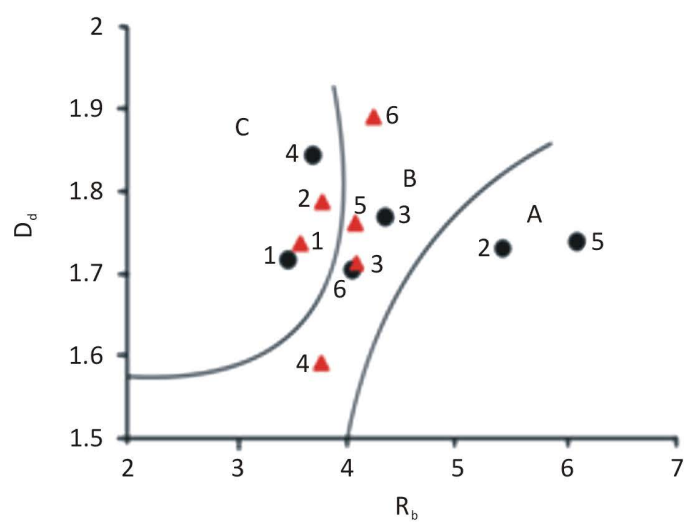

$A=$ Low flood $B=$ Intermediate flood $C=$ High flood

- Dip slope watersheds

1-W.Aub Tarfa 2 2-W.Aub Tarfa 1 3-W.el Batra 4-W.Tawayil el Hamd

5-W.Huseinan 6-W.Wuheida

A Faulted-erosional slope watersheds

1-W.Rabigh 2-W.Hafir 3-W.Ghafir 4-W.Jaded 5-W.Hanout 6-W.Jamam

(a)

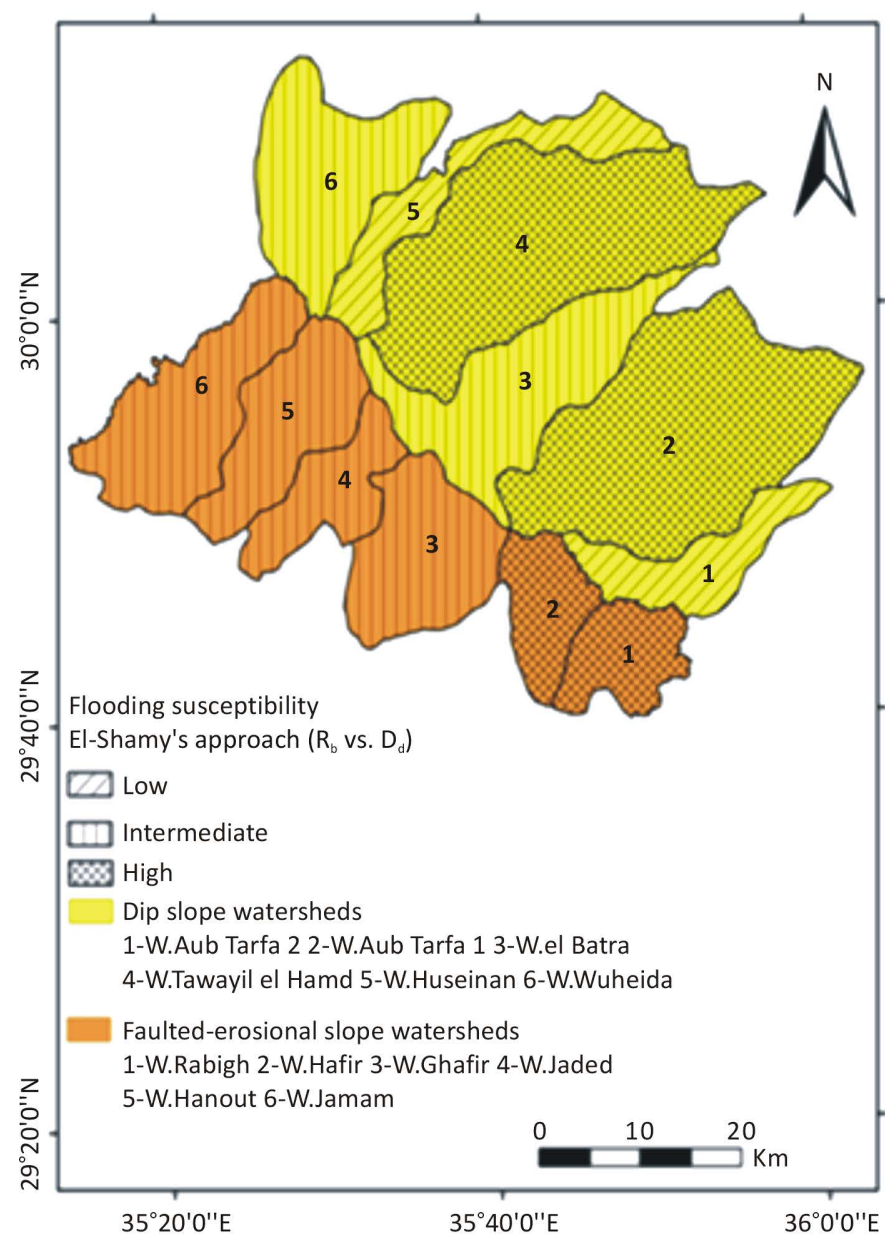

(b)

Figure 12. Flooding susceptibility (a) \& (b) based on El-Shamy's approach $\left(\mathrm{R}_{\mathrm{b}}\right.$ vs. $\left.\mathrm{D}_{\mathrm{d}}\right)$. 


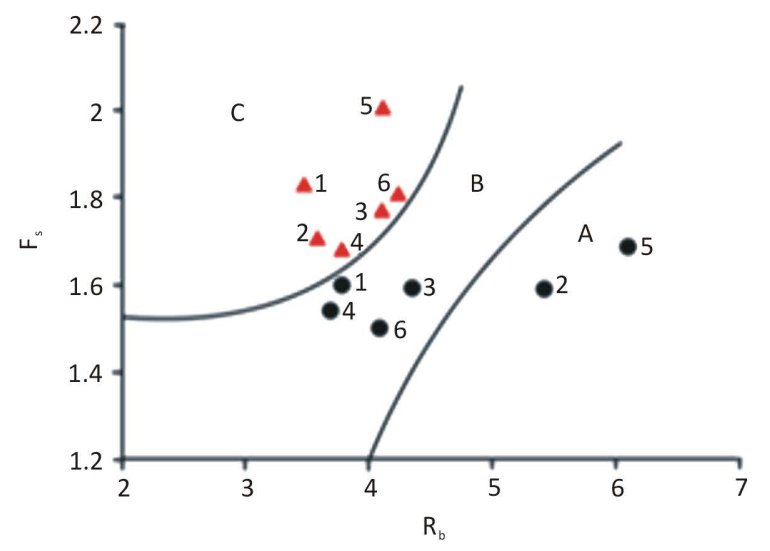

$A=$ Low flood $B=$ Intermediate flood $C=$ High flood

- Dip slope watersheds

1-W.Aub Tarfa 2 2-W.Aub Tarfa 13 -W.el Batra 4-W.Tawayil el Hamd 5-W.Huseinan 6-W.Wuheida

$\Delta$ Faulted-erosional slope watersheds

1-W.Rabigh 2-W.Hafir 3-W.Ghafir 4-W.Jaded 5-W.Hanout 6-W.Jamam

(a)

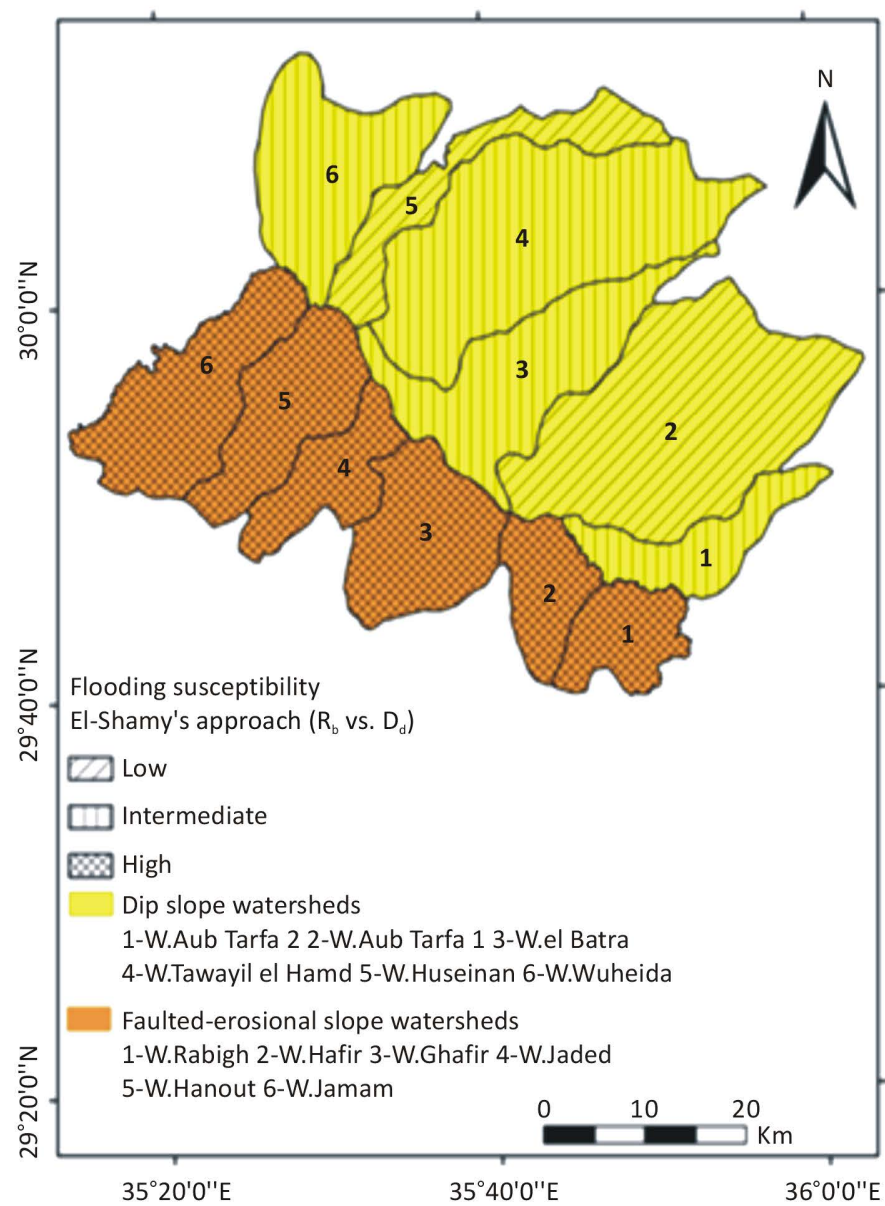

(b)

Figure 13. Flooding susceptibility (a) \& (b) according to El-Shamy's approach $\left(\mathrm{R}_{\mathrm{b}}\right.$ vs. $\left.\mathrm{F}_{\mathrm{s}}\right)$. 


\section{Conclusions}

Morphometric analysis carried out for Ras En Naqb watersheds confirms the presence of two catchment categories: the dip slope catchments and, the faulted-erosinal watersheds. A pronounced variation exists in the geomorphometric parametrers characterizing both categories. Drainage density $\left(D_{d}\right)$, relief ratio $\left(R_{r}\right)$, elongation ratio $\left(R_{e}\right)$, circularity ration $\left(R_{c}\right)$ and ruggedness number $\left(R_{n}\right)$ vary considerably. High values of mean bifurcation ratio $\left(\mathrm{R}_{\mathrm{bm}}\right)$ indicate the structural and lithological control on drainage network development across the Ras En Naqb escarpment. The variation in stream length ratio is attributed to variation in morphological characteristics of watersheds especially slope and topography. A dendritic drainage pattern dominated the dip slopes, whereas a trellised pattern characterized the faulted-erosional slopes. Channels extension by head-erosion along both terrain units of the escarpment is supported by uplifting of the scarp zone. The Ras En Nagb escarpment is well drained by the 12 watersheds which produced undulating-rolling terrain on the dip slopes, and rugged-steep topography and deeply incised wadis on the faulted-erosional slopes. The catchments of the dip slopes are more elongated, whereas the watersheds of the faulted-erosional slopes are less elongated, and approach the oval category. Values of hypsometric integral (Hi) range from 0.70 (W.Hanout, faulted-erosional slope catchment) to 0.92 (W.Huseinan, dip slope catchment), which indicates that the Ras En Naqb watersheds are at a youth-age stage of geomorphic development.

The relationship between $R_{b}$ and $D_{d}$ reveals that watersheds nos. 1 (W.Rabigh) and 2 (W.Hafir) of the faultederosional slopes are classified as with high susceptibility to flooding, whereas, four watersheds (Nos. 3, 4, 5, and 6) are categorized under intermediate flooding susceptibility. By contrast, watersheds Nos. 1 (W.Abu Tarfa 2) and 4 (W.Tawayil el Hamd) of the dip slopes are designated of high flooding potential. Similarly, watersheds Nos. 3 and 6 are denominated of intermediate susceptibility to flooding. However, the relationship between $R_{b}$ and $F_{s}$ provided more consistent results of flood hazard susceptibility. In this regard, all the six faulted-erosional slope watersheds are classified as catchments of high flooding susceptibility, while four dip slope watersheds are categorized under intermediate flooding liability. It can be concluded that ten watersheds (83.3\%) are classified under high and intermediate flooding susceptibility, and the faulted-erosional slope watersheds are more hazardous in terms of flooding. Thus, the protection of Ma'an city, El Jafr rural Bedouin settlements, and AmmanAqaba highway from recurrent flooding is essential to ensure future sustainable development in the area under consideration. Exceptional recurrent heavy rain storms, morphometric characteristics of drainage networks, pronounced/sharp morphology, and poor land cover, are the most important factors initiating flash floods in the Ras En Naqb area.

\section{References}

[1] Chorley, R. (1971) The Drainage Basin as the Fundamental Geomorphic Unit. In: Chorley, R., Ed., Introduction to Fluvial Processes, Methuen and Co. Ltd., London, 30-32.

[2] Strahler, A.N. (1952) Dynamic Basis of Geomorphology. Geological Society of America Bulletin, 63, 923-938. http://dx.doi.org/10.1130/0016-7606(1952)63[923:DBOG]2.0.CO;2

[3] Strahler, A.N. (1964) Quantitative Geomorphology of Drainage Basinsand Channel Networks. In: Chow, V.T., Ed., Handbook of Applied Hydrology, McGraw Hill, New York, Section 4-11.

[4] Chow, V.T. Ed. (1964) Handbook of Applied Hydrology. McGraw Hill, New York.

[5] Gregory, K.J. and Walling, D.E. (1973) Drainage Basin Form and Process: A Geomorphological Approach. Wiley, New York.

[6] Mesa, L.M. (2006) Morphometric Analysis of a Subtropical Andean Basin (Tucuman, Argentina). Environmental Geology, 50, 1235-1242. http://dx.doi.org/10.1007/s00254-006-0297-y

[7] Horton, R. (1945) Erosional Development of Streams and Their Drainage Basins: Hydrological Approach to Quantitative Morphology. Geological Society of America Bulletin, 56, 275-370. http://dx.doi.org/10.1130/0016-7606(1945)56[275:EDOSAT]2.0.CO;2

[8] Schumm, S.A. (1956) Evaluation of Drainage System and Slopes in Badlands at Perth Amboy, New Jersey. Geological Society of America Bulletin, 67, 597-646. http://dx.doi.org/10.1130/0016-7606(1956)67[597:EODSAS]2.0.CO;2

[9] Hadley, R. and Schumm, S. (1961) Sediment Sources and Drainage Basin Characteristics in Upper Cheyenne River Basin. US Geological Survey Water-Supply Paper 153-B, Washington DC, 198.

[10] Strahler, A.N. (1957) Quantitative Analysis of Watershed Geomorphology. Transactions, American Geophysical Union, 138, 913-920. http://dx.doi.org/10.1029/TR038i006p00913 
[11] Strahler, A.N. (1956) Quntitative Slope Analysis. Geological Society of America Bulletin, 67, 571-596. http://dx.doi.org/10.1130/0016-7606(1956)67[571:QSA]2.0.CO;2

[12] Mather, P. and Doornkamp, J. (1970) Multivariate Analysis in Geography. Transactions of the Institute of British Geographers, 51, 163-187. http://dx.doi.org/10.2307/621768

[13] Arnous, M., Aboulela, H. and Green, D. (2011) Geo-Environmental Hazard Assessment of the North Western Gulf of Suez, Egypt. Journal of Coastal Conservation, 15, 37-50. http://dx.doi.org/10.1007/s11852-010-0118-z

[14] Angillieri, M.Y.E. (2008) Morphometric Analysis of Colanguil River Basin and Flash Flood Hazard, San Juan, Argentina. Environmental Geology, 55, 107-111. http://dx.doi.org/10.1007/s00254-007-0969-2

[15] Patel, D., Dholakia, M., Naresh, N. and Srivastava, P. (2012) Water Harvesting Structure Positioning by Using GeoVisualization Concept and Prioritization of Mini Watersheds through Morphometric Analysis in the Lower Tapi Basin. Journal of the Indian Society of Remote Sensing, 40, 299-312. http://dx.doi.org/10.1007/s12524-011-0147-6

[16] Abdul Rahman, S.S., Abdul Ajeez, S.A. and Jegankumar, R. (2015) Prioritization of Sub Watershed Based on Morphometric Characteristics Using Fuzzy Analytical Hierarchy Process and Geographical Information System-A Study of Kalla Watershed, Tamil Nadu. Aquatic Procedia, 4, 1322-1330. http://dx.doi.org/10.1016/j.aqpro.2015.02.172

[17] Javed, A., Khanday, M.Y. and Ahmad, R. (2009) Prioritization of Sub-Watersheds Based on Morphometric and Land Use Analysis in Guna District (MP): A Remote Sensing and GIS Based Approach. Journal of the Indian Society of Remote Sensing, 37, 261-274. http://dx.doi.org/10.1007/s12524-009-0016-8

[18] Javed, A., Khanday, M.Y. and Rais, S. (2011) Watershed Prioritization Using Morphometric and Land Use/Land Cover Parameters: A Remote Sensing and GIS Based Approach. Journal of the Geological Society of India, 78, 63-75. http://dx.doi.org/10.1007/s12594-011-0068-6

[19] Farhan, Y. and Anaba, O. (2016) A Remote Sensing and GIS Approach for Prioritization of Wadi Shueib Mini-Watersheds(Central Jordan) Based on Morphometric and Soil Erosion Susceptibility Analysis. Journal of Geographic Information System, 8, 1-19. http://dx.doi.org/10.4236/jgis.2016.81001

[20] Sreedevi, P.D., Sreekanth, P.D., Khan, H.H and Ahmad, S. (2013) Drainage Morphometry and Its Influence on Hydrology in an Semi Arid Region: Using SRTM Data and GIS. Environmental Earth Sciences, 70, 839-848. http://dx.doi.org/10.1007/s12665-012-2172-3

[21] Prasad, R.K., Mondal, N.C., Banerjee, P., Nandakumar, M.V. and Singh, V.S. (2008) Deciphering Potential Groundwater Zone in Hard Rock through the Application of GIS. Environmental Geology, 55, 467-475. http://dx.doi.org/10.1007/s00254-007-0992-3

[22] El-Shamey, I. (1992) Recent Recharge and Flash Flooding Opportunities in the Eastern Desert, Egypt. Annals of the Geological Survey of Egypt, 18, 323-334.

[23] Beheiry, S.A. (1972) Desert Landscape in Southern Jordan. Faculty of Arts Journal (University of Jordan, Amman), 3, 5-31.

[24] De Vaumas, E. (1961) Structure et Morphologie du Proche-Orien. Revue de Geographie Alpine, 49, $225-274$. http://dx.doi.org/10.3406/rga.1961.1984

[25] Parker, D.H. (1970) The Hydrology of the Mesozoic-Cainozoic Aquifers of the Western Highlands and Plateau of East Jordan. FAO, AGL: SF/JOR, Tech Report 2, Rome.

[26] Bender, F. (1975) Geology of the Arabian Peninsula: Jordan. Geological Survey Professional Paper 560-I, Washington DC.

[27] Horowitz, A. (1979) The Quaternary of Israel. Academic Press, London.

[28] Osborn, G. (1985) Evolution of the Cenozoic Inselberg Landscape of Southwestern Jordan. Palaeogeography, Palaeoclimatology, Palaeoecology, 49, 1-23. http://dx.doi.org/10.1016/0031-0182(85)90002-1

[29] Lloyd, J.W. (1969) The Hydrology of the Southern Desert of Jordan UN Development Program/Food and Agriculture Organization of UN: Investigation of the Sandstone Aquifers of East Jordan. Technical Report 1, Rome.

[30] Osborn, G. and Duford, J.M. (1981) Geomorphological Processes in the Inselberg Region of Southwestern Jordan. Palestine Exploration Quarterly, 113, 1-17. http://dx.doi.org/10.1179/peq.1981.113.1.1

[31] Goudie, A., Migon, P., Allison, R. and Rosser, J.S. (2002) Sandstone Geomorphology of the Al-Quwayra Area of South Jordan. Zeitschrift fur Geomorphologie, 46, 365-390.

[32] Farhan, Y. and Anbar, A. (2014) Fragile Landscape: Impact and Consequences of May 2014 Flash-Flood Disaster in the Aqaba Area, Southern Jordan. Research Journal of Environment and Earth Sciences, 6, 451-465.

[33] Farhan, Y. (2014) Geomorphological Evaluation for Urban Development Using Remote Sensing and GIS, Southern Coast of Aqaba, Jordan. Journal of Environment and Earth Sciences, 4, 104-118.

[34] NASA’s Earth Observing Data and Informtion System (2014) EOSDIS-Data Specification. EOSDIS-Earthdata Web- 
site. http://www.jspacesystems.or.jp/ersdac/GDEM2/E/4.html

[35] ASTER GDEM Validation Ream (2014) ASTER Global Digital Elevation Model Version 2-Summary of Validation Results. https://www.jspacesystems.or.jp/ersdac/GDEM/ver2Validation/Summary GDEM2validation report_final.pdf.

[36] Al-Fugara, A. (2015) Comparison and Validation of the Recent Freely Available DEMs over Part of the Earth's Lowest Elevation Area: Dead Sea, Jordan. International Journal of Geosciences, 6, 1221-1232. http://dx.doi.org/10.4236/ijg.2015.611096

[37] Ouerghi, S., ELsheikh, R.A., Achour, H. and Bouazi, S. (2015) Evaluation and Validation of Recent Freely-Available ASTER-GDEM V.2, SRTM V.4.1 and the DEM Derived From Topographic Map over SW Grombalia (Test Area) in North East of Tunisia. Journal of Geographic Information System, 7, 266-279. http://dx.doi.org/10.4236/jgis.2015.73021

[38] Strahler, A.N. (1952) Hypsometric (Area Altitude) Analysis of Erosional Topography. Geological Society of America Bulletin, 63, 1117-1142. http://dx.doi.org/10.1130/0016-7606(1952)63[1117:HAAOET]2.0.CO;2

[39] Al-Bolooshi, A. (1997) Geomorphology of Ras En Naqb Escarpment, Southern Jordan, MA. Dissertation, University of Jordan, Amman.

[40] Chopra, R., Dhiman, D. and Sharma, K. (2005) Morphometric Analysis of Sub-Watersheds in Gurdaspur District, Punjab Using Remote Sensing and GIS Techniques. Journal of the Indian Society of Remote Sensing, 33, 531-539. http://dx.doi.org/10.1007/BF02990738

[41] Youssef, A., Pradhan, B. and Hassan, A. (2011) Flash Flood Risk Estimation along the St. Katherine Road, Southern Sinai, Egypt Using GIS Based Morphometry and Satellite Imagery. Environmental Earth Sciences, 62, 611-623. http://dx.doi.org/10.1007/s12665-010-0551-1

[42] Kochel, R.C. (1988) Geomorphic Impact of Large Floods: Review and New Perspectives on Magnitude and Frequency. In: Baker, V., Kochel, R. and Patton, P., Eds., Flood Geomorphology, Wiley, New York, 169-187.

[43] Tucker, G.E. and Bras, R.L. (1998) Hillslope Processes, Drainage Density, and Landscape Morphology. Water Resources Research, 34, 2751-2764. http://dx.doi.org/10.1029/98WR01474

[44] Magesh, N.S., Chandrasekar, N. and Soundranayagam, J.P. (2011) Morphometric Evaluation of Papanasam and Manimuthar Watersheds, Part of Western Ghats, Tirunelveli District, Tamil Nadu, India-A GIS Approach. Environmental Earth Sciences, 64, 373-381. http://dx.doi.org/10.1007/s12665-010-0860-4

[45] Central Water Authority (1966) Hydrolic Division. Floods in Southern Jordan on 11 March 1966, Amman, Jordan.

[46] Murphy, B. (2010) Hydrologic Analysis for a Hyper-Arid Region in the Middle East. In: Potter, J.W. and Frevert, D.K., Eds., Watershed Management: Innovations in Watershed Management under Land Use and Climate Change, ASCE, 1238-1247. http://dx.doi.org/10.1061/41143(394)111

[47] Singh, S. and Singh, M.C. (1997) Morphometric Analysis of Kanhar River Basin. National Geographical Journal of India, 43, 31-43.

[48] Ivanova, E., Nedkov, R., Ivanova, I. and Radeva, K. (2012) Morpho-Hydrographic Analysis of Black Sea Catchment Area in Bulgaria. Procedia Environmental Sciences, 14, 143-153. http://dx.doi.org/10.1016/j.proenv.2012.03.014

[49] Chorely, R., Donald, M. and Pogorzelsk, H. (1957) A New Standard for Estimating Drainage Basin Shape. American Journal of Science, 255, 138-141. http://dx.doi.org/10.2475/ajs.255.2.138

[50] Miller, V.C. (1953) A Quantitative Geomorphologic Study of Drainage Basin Characteristics in the Clinch Mountain Area, Virginia and Tennessee. Project NR 389042, Tech Report, Columbia University Department of Geology, ONR Geography Branch, New York.

[51] Ward, R.C. and Robinson, M. (2000) Principles of Hydrology. UK McGraw-Hill, Maidenhead.

[52] Smith, K.G. (1950) Standard for Grading Texture and Erosional Topography. American Journal of Science, 248, 655668. http://dx.doi.org/10.2475/ajs.248.9.655

[53] Markose, V., Dinesh, A. and Jayappa, K. (2014) Quantitative Analysis of Morphometric Parameters of Kali River Basin, Southern India, Using Bearing Azimuth and Drainage (bAd) Calculator and GIS. Environmental Earth Sciences, 72, 2887-2903. http://dx.doi.org/10.1007/s12665-014-3193-x

[54] Burdon, D. (1959) Handbook of the Geology of Jordan. Benham \& Co., Colchester.

[55] Faniran, A. (1968) The Index of Drainage Intensity-A Provisional New Drainage Factor. Australian Journal of Science, 31, 328-330.

[56] Singh, S. and Dubey, A. (1994) Geo-Environmental Planning of Watersheds in India. Chugh Publications, Allahabad, 28-69.

[57] Farhan, Y., Anbar, A., Enaba, O. and Al-Shaikh, N. (2015) Quantitative Analysis of Geomorphometric Parameters of Wadi Kerak, Jordan Using Remote Sensing and GIS. Journal of Water Resources and Protection, 7, 456-175. 
http://dx.doi.org/10.4236/jwarp.2015.76037

[58] Markose, V. and Jayappa, K. (2011) Hypsometric Analysis of Kali River Basin, Karnataka, India, Using Geographic Information System. Geocarto International, 26, 553-568. http://dx.doi.org/10.1080/10106049.2011.608438

[59] Singh, O., Sarangi, A. and Sharma, M.C. (2008) Hypsometric Integral Estimation Methods and its Relevance on Erosion Status of Northwestern Lesser Himalayan Watersheds. Water Resources Management, 22, 1545-1560. http://dx.doi.org/10.1007/s11269-008-9242-z

[60] Bishop, M.P., Shroder, J.F., Bonk, R. and Olsenholler, J. (2002) Geomorphic Changes in High Mountains: A Western Himalaya Perspective. Global and Planetary Change, 32, 311-329. http://dx.doi.org/10.1016/S0921-8181(02)00073-5

[61] Hurtrez, J., Lucazean, F. and Avouac, J. (1999) Investigation of the Relationship between Basin Morphometry, Tectonic Uplift and Denudation from the Study of an Active Fold Belt in Siwalik Hills, Central Nepal. Journal of Geophysical Research B, 104, 12779-12796. http://dx.doi.org/10.1029/1998JB900098

[62] Weissel, J., Pratson, L. and Malinverno, A. (1994) The Length-Scaling Properties of Topography. Journal of Geophysical Research, 99, 13997-14012. http://dx.doi.org/10.1029/94JB00130 